\title{
Comparison of diagenetic and low-grade metamorphic evolution of chlorite in associated metapelites and metabasites: an integrated TEM and XRD study
}

\author{
P. ÁRKAI, ${ }^{1}$ M. P. MATA, ${ }^{2}$ G. GIORGETTI, ${ }^{2}$ D. R. PEACOR ${ }^{2}$ AND M. TÓTH ${ }^{1}$ \\ ${ }^{1}$ Laboratory for Geochemical Research, Hungarian Academy of Sciences, H-1112 Budapest, Budaoersi út 45, Hungary \\ (arkai@sparc.core.hu) \\ ${ }^{2}$ Department of Geological Sciences, The University of Michigan, Ann Arbor, MI 48109, USA
}

\begin{abstract}
Chlorite is a common sheet silicate that occurs in various lithologies over a wide grade range involving diagenesis and low-grade metamorphism. Thus, the reaction progress of chlorite offers a unique opportunity for direct correlation of zonal classification of metasedimentary rocks based on illite crystallinity with metabasite mineral facies. To provide such correlation, chlorite crystallinity indices, apparent mean crystallite sizes and lattice strains, crystallite size distributions and compositions of chlorite from coexisting metapelites and metabasites were determined by X-ray diffraction (XRD), scanning electron microscopy (SEM), transmission electron microscopy (TEM), analytical electron microscopy (AEM) and electron microprobe (EMP) methods. Samples were from Palaeozoic and Mesozoic formations of the Bükkium (innermost Western Carpathians, Hungary) that underwent Alpine (Cretaceous) orogenic metamorphism. Metapelites range in grade from late diagenesis to epizone, whereas metabasites vary from prehnitepumpellyite through pumpellyite-actinolite to greenschist facies.

Despite significant differences in composition, mineral assemblages and textures, reaction progress, as measured in part by chlorite crystallinity, in metapelites paralleled that in metabasites. Chlorite crystallinity and mean crystallite size increase and the proportion of mixed layers in chlorite decreases, whereas the calculated lattice strain does not change significantly with increasing metamorphic grade. Similar trends, but (especially at higher grades) significant differences, were found in mean crystallite size values using various methods for XRD line profile analyses. The increase in crystallite size with increasing grade was demonstrated also by direct TEM measurements on ion-milled whole-rock samples, but with a larger scatter of data at higher grades. In spite of the different kinds of mixed layering in chlorite (Mg-rich smectitic, mostly random, local corrensite-like units in metabasites, and Fe-rich berthierine and dioctahedral smectite in metapelites), XRD-calculated and TEM-measured parameters were found to be reliable tools for measuring reaction progress and metamorphic grade of the same degree in both lithotypes.
\end{abstract}

Key words: chlorite crystallinity; crystallite size; low-temperature metamorphism; metabasite; metapelite.

\section{INTRODUCTION}

The transitional realm between diagenesis and metamorphism is characterized by several features that strongly hinder the accurate determination of grade (temperature) of transformation. Significant factors include the general lack of stable thermodynamic equilibrium assemblages in common rock types, the restriction of key mineral assemblages to relatively narrow ranges of bulk rock compositions (basic to intermediate igneous rocks and their sedimentary derivatives), and an extremely narrow range of fluid compositions (fluids with very low $\mathrm{CO}_{2}$ and high $\mathrm{H}_{2} \mathrm{O}$ contents). These relations have been central to studies of changing mineral textures, compositions and structures as a function of geological parameters in attempts to correlate such features directly with grade (for recent reviews, see Frey, 1987; Teichmüller, 1987; Merriman \& Frey, 1999; Merriman \& Peacor, 1999; Robinson \& Merriman, 1999).
Long after the introduction and widespread successful application of the concept of illite crystallinity (abbreviated as IC or KI, see also Kübler, 1967, 1968), similar prograde relations were studied for chlorite and its precursor minerals, beginning in the late 1980s. Early applications of the chlorite crystallinity (ChC) parameter were briefly reviewed by Frey (1987). In correlating the prograde evolution of illite-muscovite and chlorite, Árkai (1991) found relatively strong $(r=$ 0.75-0.85) positive linear correlations between IC and ChC. Árkai also gave a generalized scheme for the correlation of $\mathrm{IC}, \mathrm{ChC}$, vitrinite reflectance (VR), conodont colour (CAI) scales and metabasite mineral facies. As is the case for changes in values of IC, a decrease in mixed layering, increase in crystallite size and decrease in lattice strain were considered to be the main factors affecting ChC. Yang \& Hesse (1991) explained the consistent behaviour of IC and $\mathrm{ChC}(001)$ in anchizonal rocks in terms of decreasing amounts of expandable mixed layers, whereas $\mathrm{ChC}(002)$ reflects 
chlorite crystallinity, sensu stricto, i.e. for pure chlorite with no mixed layering. Árkai et al. (1995b) confirmed the applicability of $\mathrm{ChC}$ for monitoring diagenetic through incipient metamorphic grades of fine-grained clastic metasediments using a large $(n=238)$ sample set from Palaeozoic rocks of the Southern Alps.

Essene \& Peacor (1995) and Merriman \& Peacor (1999) reviewed aspects of clay mineral thermometers, emphasizing that variables which affect IC and $\mathrm{ChC}$, such as the extent of mixed layering, crystallite size and amount of lattice strain in low-grade rocks, are not functions of stable thermodynamic equilibrium. Rather, they are measures of the degree to which dioctahedral and trioctahedral minerals have approached the stable thermodynamic state as generally attained in epizonal rocks. Because 'grade' is a direct function of temperature, and because IC and $\mathrm{ChC}$ are measures of states of metastable equilibrium, the term 'reaction progress' was inferred to be more appropriate as an expression of the state of dioctahedral and trioctahedral minerals in low-grade rocks.

The continuous and discontinuous structural and chemical changes of mafic phyllosilicates occurring in metabasites have been extensively studied (for recent reviews, see Árkai \& Sadek Ghabrial, 1997; Alt, 1999; Robinson \& Bevins, 1999; Schiffman \& Day, 1999). Árkai \& Sadek Ghabrial (1997) demonstrated that, as in the case of metasediments, values of $\mathrm{ChC}$ can also be applied to determine relative differences in grades of low-grade metabasites. Comparison of electron microprobe (EMP) and X-ray diffraction (XRD) data showed that $\mathrm{ChC}$ is controlled mainly by the decreasing amounts of contaminants in chlorite (mixed-layered components or discrete, intergrown phases, primarily smectite-like).

$\mathrm{ChC}$ is therefore a unique tool for direct correlation of reaction progress in metasediments and metabasites with grade as determined by mineral facies. However, because it occurs commonly in various lithologies over a wide range of grade involving diagenesis through low-temperature metamorphism, it may also be used to determine reaction progress of certain common rock types for which none of the existing petrogenetic schemes can now be applied. Such rock types include, for example, carbonate-rich meta-igneous rocks and their clastic equivalents (tuffs, tuffites, graywackes) devoid of key mineral assemblages, illite and organic matter. A critical factor in the correlation of chlorite crystallinities determined in different rock types, however, is the degree to which different rock compositions (e.g. metabasites vs. metapelites) and textures (crystallite sizes) are the cause of differences in the degree of reaction progress of chlorite as a function of grade and other geological factors. Because $\mathrm{ChC}$ is principally a function of crystallite size, for example, and because the small crystallites found at low grades are not in a state of stable equilibrium (Merriman \& Peacor, 1999), variations in composition and structure may be expected to result in different degrees of reaction progress in different rock types subjected to the same grades of metamorphism.

Therefore, in order to assess the mineralogical meaning of $\mathrm{ChC}$, and to evaluate its value as a petrogenetic tool, integrated XRD, scanning (SEM), analytical (AEM) and high-resolution (scanning) transmission (STEM and TEM) electron microscopy and EMP investigations of chlorite were carried out on a selected set of coexisting pairs of metapelites and metabasites which had been previously studied by XRD and EMP by Árkai \& Sadek Ghabrial (1997). The aim of the present study was to determine the similarities and differences between reaction progress in trioctahedral phyllosilicates, principally chlorite, from these two contrasting lithotypes, by comparing their $\mathrm{ChC}$ indices, apparent mean crystallite sizes and lattice strain values as calculated from XRD data, TEM-determined mean crystallite sizes and size distributions and chemical compositions determined by AEM and EMP. This report emphasizes the relation between the evolution of trioctahedral clays, principally chlorite, and those factors principally affecting values of ChC. The evolution of mineralogical relations as primarily observed by TEM will be described in a separate contribution, and only a brief description of such relations is therefore given below.

\section{MATERIALS}

A series of samples was collected from the innermost tectonic units of the Western Carpathians, with metabasite and metapelite samples obtained in pairs, so that conditions of low-grade metamorphism would be as similar as possible (Fig. 1). As shown by palaeogeographic reconstructions of Kovács (1989) and Kovács et al. (1996-97), the South Gemer Unit, to which the Darnó Hill and Szarvaskö complexes

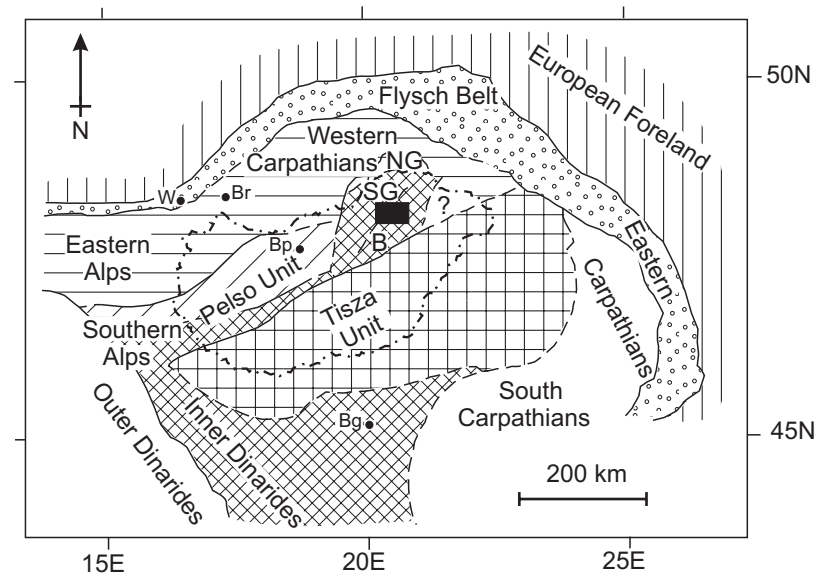

Fig. 1. Tectonic setting of the Bükkium and the South Gemer Unit within the Alp-Carpathian-Dinaric framework. Dasheddotted line indicates the state boundary of Hungary.

Abbreviations: B, Bükkium; SG, South Gemer Unit; NG,

North Gemer Unit; Bp, Budapest; W, Vienna; Br, Bratislava; Bg, Beograd. 
belong, contains fragments of Middle Triassic and Middle-Upper Jurassic oceanic crust, as indicated by the incomplete, dismembered ophiolitic formations of the so-called Axios-Vardar-Meliata oceanic branch of the Neothetys. The mobile, outer shelf-type Bükkium (i.e. the Palaeozoic and Mesozoic formations of the north-east Hungarian Bükk, Uppony and Szendrö Mountains) comprised the southern (African) border of this oceanic basin. Recent plate tectonic reconstructions restore the Bükkium to the north-western part of the Inner Dinarides, implying juxtaposition by mostly meso-Alpine, large-scale horizontal displacements (Kovács, 1989; Kovács et al., 1996-97).

Figure 2 displays the main geological and tectonic features of the Bükk and Uppony Mountains. The rock types, metamorphic grades, primary and metamorphic ages and localities are given in Table 1. The following brief sample descriptions refer to the geological and tectonic summary of Kovács et al. (1996-97) as well as the metamorphic petrogenetic results of Árkai (1983, 1991), Árkai et al. (1995a) and Sadek Ghabrial et al. (1996).

The Mesozoic section of the Darnó Hills (southwestern end of the Bükk Mountains), which is separated by a Cenozoic strike-slip fault zone from other parts of the mountains and the Szarvaskö complex (=Szarvaskö-Mónosbél nappe) that forms the uppermost tectonic unit of the Bükk Mountains, belongs to the oceanic Meliata Unit. In the Darnó Hills, the Middle Triassic pillow basalts display oceanfloor hydrothermal metamorphism with prehnitepumpellyite facies assemblages, whereas the interbedded pelitic, cherty and marly sediments underwent diagenesis. The Szarvaskö complex consists of a Jurassic, incomplete, dismembered, MORB-type, ophiolite-like ultrabasic-basic-(acidic) sequence (Downes et al., 1990) and clastic sedimentary rocks. The Jurassic sediments under- and overlie the magmatic complex and are in contact with sheeted-dyke and cumulategabbro members. With the exception of the remnants of high- $T /$ low- $P$ hydrothermal metamorphism of gabbros, the meta-igneous rocks of the Szarvaskö complex display prehnite-pumpellyite facies assemblages that are compatible with regional (dynamothermal) modification of the clastic sedimentary units with resultant variation in grade from late or deep diagenesis to the lower anchizone.

The eastern and northern parts of the Bükk Mountains form the Bükk Parautochthon, i.e. the lowest tectonic unit of the mountains. The Bükk Parautochthon consists of Middle Carboniferous to Late Jurassic sequences in several tectonic slices. In

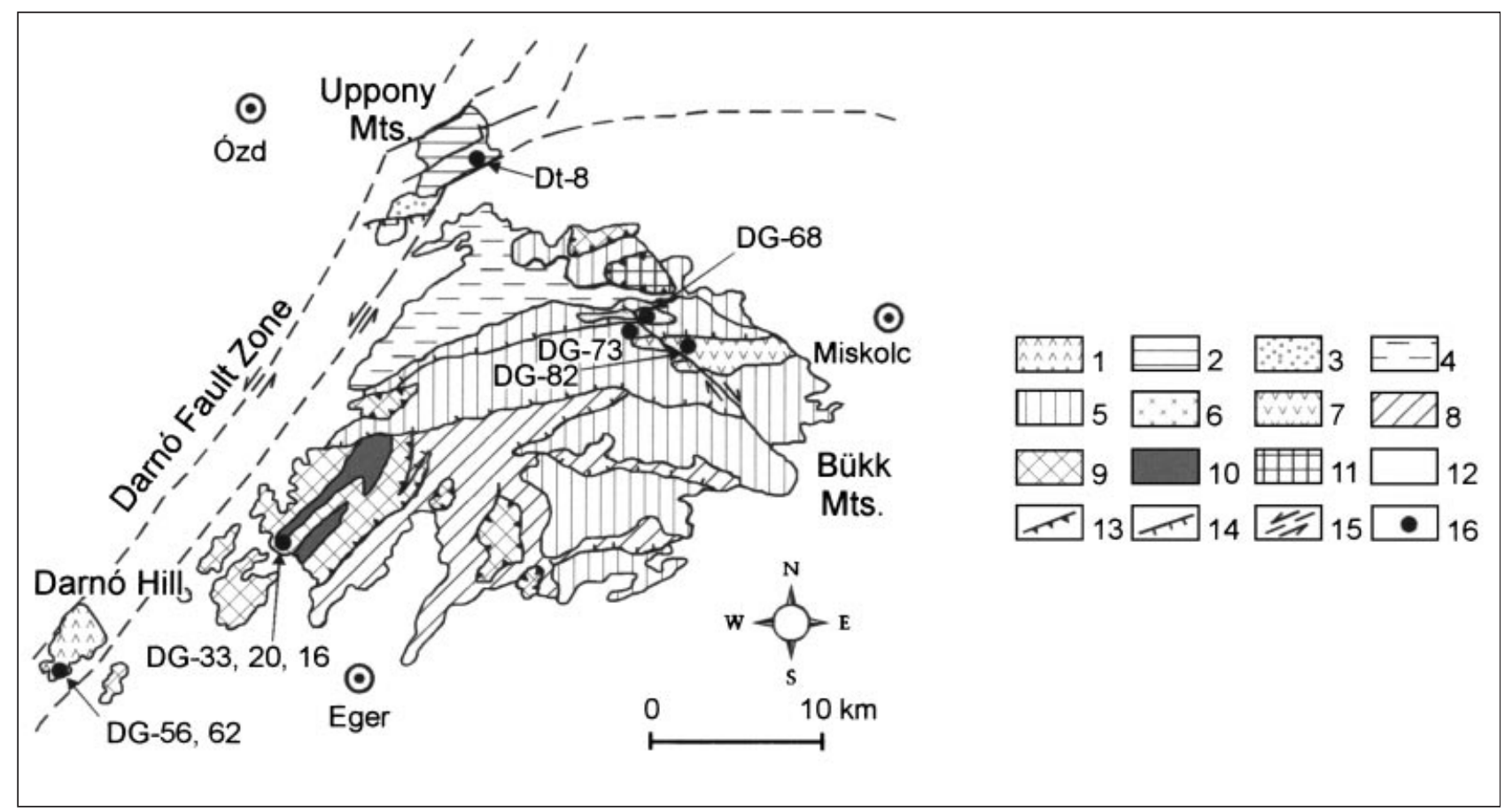

Fig. 2. Geological sketch map of the Bükk and Uppony Mountains after Kovács (1989), simplified, with sample locations. Key: 1 , Meliaticum: Triassic and Jurassic metabasites and metasediments of the Darnó Hill, SW Bükk Mountains; 2, Uppony Palaeozoic (Ordovician(?)-Middle Carboniferous); 3, Gosau-type Senonian conglomerates in the Uppony Mountains; 4-8, Bükk Parautochthon: 4, Upper Palaeozoic; 5, Triassic sedimentary formations; 6, Ladinian meta-andesite and tuffs; 7, Carnian metabasalt, metarhyolite and their tuffs; 8, Jurassic sedimentary rocks; 9-10, Szarvaskö-Mónosbél nappe: 9, Jurassic sedimentary rocks; 10, Jurassic incomplete, dismembered ophiolite complex; 11, Kisfennsík ('Little Plateau') nappe (Triassic); 12, Cenozoic; 13, nappe boundary; 14, major imbrications within nappes; 15 , strike-slip fault; 16, location of samples. 
Table 1. Rock samples investigated.

\begin{tabular}{|c|c|c|c|c|c|c|}
\hline Sample & Rock type & $\begin{array}{l}\text { Metamorphic facies, } \\
\text { zone }\end{array}$ & $\begin{array}{l}\text { Stratigraphic } \\
\text { age }\end{array}$ & $\begin{array}{l}\text { Metamorphic } \\
\text { age }\end{array}$ & Locality & Tectonic unit \\
\hline DG-56 & $\begin{array}{l}\text { Metabasalt } \\
\text { (pillow lava) }\end{array}$ & $\begin{array}{l}\text { Prh-Pmp } \\
\text { (ocean floor) }\end{array}$ & $\begin{array}{l}\text { Triassic, } \\
\text { Ladinian }\end{array}$ & $\begin{array}{l}\text { Triassic(?)- } \\
\text { Jurassic(?) }\end{array}$ & Darnó Hill & Meliata \\
\hline DG-62 & Shale & Diagenetic IC & $\begin{array}{l}\text { Triassic, } \\
\text { Ladinian }\end{array}$ & $\begin{array}{l}\text { Triassic(?)- } \\
\text { Jurassic(?) }\end{array}$ & Darnó Hill & Meliata \\
\hline DG-33 & $\begin{array}{l}\text { Metabasalt } \\
\text { (pillow lava) }\end{array}$ & $\begin{array}{l}\text { Prh-Pmp } \\
\text { (reg. dynamothermal) }\end{array}$ & $\begin{array}{l}\text { Middle } \\
\text { Jurassic }\end{array}$ & Cretaceous & $\begin{array}{l}\text { Szarvaskö, } \\
\text { W Bükk Mts. }\end{array}$ & $\begin{array}{l}\text { Szarvaskö-Mónosbél } \\
\text { nappe, Meliata }\end{array}$ \\
\hline DG-20 & $\begin{array}{l}\text { Slate with } \\
\text { pencil cleavage }\end{array}$ & Diagenetic IC & $\begin{array}{l}\text { Middle } \\
\text { Jurassic }\end{array}$ & Cretaceous & $\begin{array}{l}\text { Szarvaskö, } \\
\text { W Bükk Mts. }\end{array}$ & $\begin{array}{l}\text { Szarvaskö-Mónosbél } \\
\text { nappe, Meliata }\end{array}$ \\
\hline DG-16 & Metadiabase & $\begin{array}{l}\text { Prh-Pmp } \\
\text { (reg. dynamothermal) }\end{array}$ & $\begin{array}{l}\text { Middle } \\
\text { Jurassic }\end{array}$ & Cretaceous & $\begin{array}{l}\text { Szarvaskö, } \\
\text { W Bükk Mts. }\end{array}$ & $\begin{array}{l}\text { Szarvaskö-Mónosbél } \\
\text { nappe, Meliata }\end{array}$ \\
\hline DG-82 & Metabasalt & $\begin{array}{l}\text { Pmp-Act } \\
\text { (reg. dynamothermal) }\end{array}$ & $\begin{array}{l}\text { Triassic, } \\
\text { Carnian }\end{array}$ & Cretaceous & $\begin{array}{l}\text { Szinva spring, } \\
\text { E Bükk Mts. }\end{array}$ & $\begin{array}{l}\text { Bükk Parautochthon, } \\
\text { Bükkium }\end{array}$ \\
\hline DG-73 & Slate & Anchizone & $\begin{array}{l}\text { Triassic, } \\
\text { Carnian }\end{array}$ & Cretaceous & $\begin{array}{l}\text { Vessö’s Valley, } \\
\text { E Bükk Mts. }\end{array}$ & $\begin{array}{l}\text { Bükk Parautochthon, } \\
\text { Bükkium }\end{array}$ \\
\hline DG-68 & Meta-andesite tuff & $\begin{array}{l}\text { Greenchist } \\
\text { (reg. dynamothermal) }\end{array}$ & $\begin{array}{l}\text { Triassic, } \\
\text { Ladinian }\end{array}$ & Cretaceous & $\begin{array}{l}\text { Lillafüred, } \\
\text { E Bükk Mts. }\end{array}$ & $\begin{array}{l}\text { Bükk Parautochthon, } \\
\text { Bükkium }\end{array}$ \\
\hline $\begin{array}{l}\text { Dt-8 } \\
(118.0 \mathrm{~m})\end{array}$ & Slate & Epizone & Silurian(?) & Cretaceous & Borehole No. Dt-8 & $\begin{array}{l}\text { Tapolcsány Unit, } \\
\text { Uppony Palaeozoic, Bükkium }\end{array}$ \\
\hline $\begin{array}{l}\text { Dt- } 8 \\
(129.5 \mathrm{~m})\end{array}$ & Metabasalt & Greenschist & Silurian(?) & Cretaceous & Borehole No. Dt- 8 & $\begin{array}{l}\text { Tapolcsány Unit, } \\
\text { Uppony Palaeozoic, Bükkium }\end{array}$ \\
\hline $\begin{array}{l}\text { Dt-8 } \\
(207.8 \mathrm{~m})\end{array}$ & Metasandstone & Epizone & Silurian(?) & Cretaceous & Borehole No. Dt-8 & $\begin{array}{l}\text { Tapolcsány Unit, } \\
\text { Uppony Palaeozoic, Bükkium }\end{array}$ \\
\hline
\end{tabular}

the eastern part of the Bükk Mountains, the Upper Triassic (Carnian) bimodal volcanic complex, with voluminous basaltic tuffs and lavas and subordinate rhyolites and tuffs, was affected by pumpellyiteactinolite facies regional metamorphism. The grade of regional metamorphism of the Middle Triassic (Ladinian) stratovolcanic formation in the Eastern Bükk, comprising lavas and predominantly finegrained pyroclastic rocks primarily of andesitic and partly basaltic compositions, reached the chlorite zone of the greenschist facies. The Carnian fine-clastic sedimentary formation that separates the abovementioned igneous units was metamorphosed under epizonal and higher anchizonal conditions.

The Uppony Mountains consist primarily of epizonal Palaeozoic formations ranging in sedimentary/ igneous ages from Ordovician(?) to Middle Carboniferous. From the variety of formations intersected by borehole Dt-8, Silurian rocks, namely epizonal slate and metasandstone intercalated with greenschist facies metabasalt, were selected for this study.

All of the Mesozoic and Palaeozoic formations of the Bükkium and the investigated nappes of the South Gemer (Meliata) Unit suffered low to transitional lowintermediate pressure-type Alpine regional metamorphism in the interval between the Upper Jurassic and Middle Cretaceous (i.e. between the eo-Hellenic and Austrian phases of the Alpine cycle).

\section{METHODS}

X-Ray powder diffraction patterns were obtained from both nonoriented and highly oriented powder mounts of whole rock and $<2 \mu \mathrm{m}$ spherical equivalent diameter (SED) size fractions. XRD data were in part used for the determination of values of illite crystallinity [IC, i.e. the full width at half-maximum (FWHM) of the first, $10 \AA$ basal reflection of illite-muscovite] and chlorite crystallinity [ChC, i.e. the FWHM values of the first $(14 \AA)$ and second $(7 \AA)$ basal reflections of chlorite, denoted as $\mathrm{ChC}(001)$ and $\mathrm{ChC}(002)$, respectively] values. The $<2 \mu \mathrm{m}$ fraction samples were prepared by methods similar to that of Kübler (1968), as described by Arkai (1991). Portions of the air-dried $<2 \mu \mathrm{m}$ fraction samples were saturated with $0.5 \mathrm{~mol}^{-1} \mathrm{MgCl}_{2}$ and, subsequently, with $1 \mathrm{~mol} \mathrm{l}^{-1} \mathrm{KCl}$ solutions. Aqueous suspensions were pipetted onto glass slides and dried at room temperature to produce highly oriented mounts having concentrations of $3 \mathrm{mg} \mathrm{cm} \mathrm{cm}^{-2}$. Air-dried (AD), $\mathrm{Mg}$-saturated and air-dried $(\mathrm{Mg}, \mathrm{AD})$ and $\mathrm{Mg}+\mathrm{K}$-saturated and air-dried $(\mathrm{Mg}+\mathrm{K}, \mathrm{AD})$ mounts were thus obtained. Ethylene glycol (EG) and glycerol (GL) solvations $\left(80^{\circ} \mathrm{C} /\right.$ overnight), as well as mounts of the $(\mathrm{Mg}+\mathrm{K}, \mathrm{AD})$ material heat treated at $490{ }^{\circ} \mathrm{C}$ for $1 \mathrm{~h}$, completed the sample preparation procedure. The XRD instrumental and measuring conditions were: Philips PW-1730 diffractometer with computerized APD system, $\mathrm{Cu} \mathrm{K}_{\alpha}$ radiation, $45 \mathrm{kV} / 35 \mathrm{~mA}$, proportional counter, graphite monochromator, divergence and detector slits of $1^{\circ}$, and collection of data with $0.02^{\circ} 2 \Theta$ steps, using time intervals of $5 \mathrm{~s}$. The calibration of IC and $\mathrm{ChC}$ values against Kübler's IC scale, where values for the anchizone range between 0.25 and $0.42^{\circ} \Delta 2 \Theta$, was made using standard rock slab series (Nos. 32, 34 \& 35) kindly provided by B. Kübler. Smaller scale variations in instrumental conditions were corrected by the repeated use of another calibrated standard rock slab series (Nos. A-1, -2 \& -3) of the Laboratory for Geochemical Research, Budapest. Applying the least-squares method, the calibration equation is:

$$
\mathrm{IC}(\text { Kübler })=1.124 \times \mathrm{IC}(\text { present work })-0.069
$$

The actual boundary ranges of $\mathrm{IC}, \mathrm{ChC}(001)$ and $\mathrm{ChC}(002)$ of the present paper, which correspond to Kübler's original anchizone, are $0.284-0.435^{\circ}, 0.309-0.390^{\circ}$ and $0.284-0.348^{\circ} \Delta 2 \Theta$, respectively (see also Árkai et al., 1995b). All of these boundary values refer to $\mathrm{AD}$ mounts.

Determination of the mean crystallite size and lattice strain from calculations based on XRD patterns may be subject to many errors; Árkai et al. $(1996,1997)$ discussed the various factors extensively. Because of these factors, calculated values can only be considered as 'apparent' mean values. In the following, crystallite size refers to the mean size of crystallites (i.e. domains that coherently scatter $\mathrm{X}$-rays) perpendicular to (001). Values were obtained by three methods.

1 The Scherrer equation (see Klug \& Alexander, 1974; for practical details, see Merriman et al., 1990).

2 The Voigt method of Langford (1978) as modified by the Philips APD-1700 software package, also referred to as the single-line 
Fourier method (in the following: Voigt method, using the DECPROF program of A. Nagy written for the Laboratory for Geochemical Research, Hungarian Academy of Sciences; for details, see Árkai et al., 1996, 1997).

3 The single-line Fourier method and the Warren-Averbach method using Krumm's (1994) WINFIT $2.1{ }^{\circledR}$ program.

Samples for STEM observation were first prepared as glassmounted thin sections cut normal to the dominant microfabric in order to optimize orientation of $\mathrm{c}^{*}$ of phyllosilicates normal to the electron beam of the scanning transmission electron microscope. Following optical and SEM observation using a Hitachi S-570 scanning electron microscope fitted with Noran energy-dispersive X-ray (EDS) and back-scattered electron (BSE) detectors, selected areas of thin sections were removed and ion milled. TEM observations were made using a Phillips CM12 scanning transmission electron microscope at The University of Michigan. All conditions of observation were as described by Merriman et al. (1990). Minerals were identified using integrated selected area electron diffraction (SAED) data, lattice fringe images, textures as defined by TEM images and EDS analyses as obtained using a Kevex Quantum EDS system. Crystal thickness measurements were made on lattice fringe images by counting regularly spaced $10 \AA$ and $14 \AA$ fringes parallel to $c^{*}$, between obvious discontinuities in the sequences of layers, as described by Merriman et al. (1990).

AEM and EMP techniques were used for the determination of chemical compositions of chlorite. Formulae were calculated from AEM spectra by using $k$ values of ion-milled standards of paragonite, muscovite, albite, clinochlore, fayalite, rhodonite and titanite, following the procedure of Jiang et al. (1990). EMP analyses were carried out with a JEOL JCXA-733 instrument equipped with three wavelength-dispersive X-ray spectrometers, using the correction method of Bence \& Albee (1968). Measuring conditions, standards and errors of quantitative analyses were the same as given by Árkai \& Sadek Ghabrial (1997).

Major element compositions of bulk rock samples were determined using a Perkin Elmer 5000 atomic absorption spectrophotometer, after digestion with lithium metaborate. In addition to the atomic absorption spectrophotometry (AAS) technique, permanganometric $(\mathrm{FeO})$, gravimetric $\left(\mathrm{SiO}_{2}, \mathrm{TiO}_{2}, \mathrm{H}_{2} \mathrm{O} \& \mathrm{P}_{2} \mathrm{O}_{5}\right)$ and volumetric $\left(\mathrm{CO}_{2}\right)$ methods were applied.

\section{RESULTS}

\section{Modal and bulk rock compositions}

Table 2 summarizes the modal compositions of the selected rock samples. The pelitic rocks of all grades comprise quartz, albite, illite-muscovite, chlorite \pm pyrite, calcite, K-feldspar and rutile. The metasandstone sample from borehole Dt-8 also con- tains minor authigenic Ba-bearing $\mathrm{K}$-feldspar, closely associated with detrital K-feldspar and albite. $\mathrm{Ca}$-clinopyroxene represents the only relic magmatic phase in the metabasites. Metabasites of all grades consist of quartz, albite, chlorite and calcite, with a variety of minerals characteristic of different grades, including $\mathrm{Ca}-\mathrm{Al}$ hydrous silicates, chlorite/smectite, actinolite and stilpnomelane. Dioctahedral micas (phengite and celadonite) are also found in some metabasites, but K-feldspar was identified in only one. Accessories include magnetite, hematite, ilmenite, titanite, apatite and rutile. Bulk rock compositions are listed in Table 3.

\section{Chlorite and illite crystallinity indices}

IC values measured on air-dried mounts of pelitic samples (Table 4) decrease from the diagenetic zone (Darnó Hill, sample DG-62; Szarvaskö complex: DG-20) to the epizone (Eastern Bükk: DG-73), whereas the slate sample from the Uppony Mountains (Dt-8 118.0m) gave a high- $T$, anchizonal, IC value. The effect of $\mathrm{Mg}$ saturation on the $10 \AA$ reflection of illite-muscovite is rather equivocal; in three cases, sharpening and, in one sample, negligible broadening of the peaks were observed. Glycolation caused sharpening of the $10 \AA$ reflection; the differences between $\mathrm{IC}_{\mathrm{AD}}$ and $\mathrm{IC}_{\mathrm{EG}}$ decrease with increasing grade.

The $\mathrm{ChC}$ values of the pelitic rocks are identical with, or slightly smaller than, those of the coexisting metabasites (Table 4). The $\mathrm{ChC}(001)$ and $\mathrm{ChC}(002)$ values measured for the pelitic rocks and associated metabasites display trends similar to that described above for the IC values of the metasediments. Thus, provisionally applying the $\mathrm{ChC}$ zone boundaries determined by Árkai et al. (1995b) for metapelites, the ChC indices of the Darnó Hill and the Szarvaskö complexes correspond to the diagenetic zone, those of the Eastern Bükk to the higher anchizone but with transition to the epizone, whereas those of the Uppony Palaeozoic fall into the epizone and to the boundary between the anchi- and epizones.

$\mathrm{Mg}$ saturation had relatively little effect on $\mathrm{ChC}(001)$

Table 2. Modal composition of whole-rock samples.

\begin{tabular}{|c|c|c|c|c|c|c|c|c|c|c|c|c|c|c|c|c|c|c|c|c|c|c|}
\hline Sample & Qtz & $\mathrm{Ab}$ & Kfs & Bafs & Ill-Ms & $\mathrm{Chl}$ & $\mathrm{Chl} / \mathrm{Sm}$ & Cpx & Act & Ep & Pmp & Prh & Stp & $\mathrm{Cal}$ & Ank & Py & Mag & $\mathrm{Hem}$ & Rt & Ilm & Ttn & Ap \\
\hline DG-56 & $\mathrm{x}$ & $\mathrm{x}$ & & & & $\mathrm{x}$ & $\mathrm{x}$ & $\mathrm{x}$ & & & $\mathrm{x}$ & $\mathrm{x}$ & & $\mathrm{x}$ & & & $\mathrm{x}$ & $\mathrm{x}$ & & $\mathrm{x}$ & $\mathrm{x}$ & $\mathrm{x}$ \\
\hline$D G-62$ & $x$ & $x$ & & & $x$ & $x$ & & & & & & & & & & $x$ & & & & & & \\
\hline DG-33 & $\mathrm{x}$ & $\mathrm{x}$ & & & $\mathrm{x}^{\mathrm{a}}$ & $\mathrm{x}$ & $\mathrm{x}$ & $\mathrm{x}$ & & $\mathrm{x}$ & $\mathrm{x}$ & $\mathrm{x}$ & & $\mathrm{x}$ & & & $\mathrm{x}$ & & & $\mathrm{x}$ & $\mathrm{x}$ & $\mathrm{x}$ \\
\hline$D G-20$ & $x$ & $x$ & & & $x$ & $x$ & & & & & & & & $x$ & & & & & & & & \\
\hline DG-16 & $\mathrm{x}$ & $\mathrm{x}$ & & & $\mathrm{x}^{\mathrm{a}}$ & $\mathrm{x}$ & & $\mathrm{x}$ & $\mathrm{x}$ & & $\mathrm{x}$ & $\mathrm{x}$ & & $\mathrm{x}$ & & & $\mathrm{x}$ & $\mathrm{x}$ & $\mathrm{x}$ & $\mathrm{x}$ & $\mathrm{x}$ & $\mathrm{x}$ \\
\hline DG-82 & $\mathrm{x}$ & $\mathrm{x}$ & & & $\mathrm{x}$ & $\mathrm{x}$ & & $\mathrm{x}$ & $\mathrm{x}$ & $\mathrm{x}$ & $\mathrm{x}$ & & & $\mathrm{x}$ & & & & $\mathrm{x}$ & & $\mathrm{x}$ & $\mathrm{x}$ & $\mathrm{x}$ \\
\hline$D G-73$ & $x$ & $x$ & & & $x$ & $x$ & $x$ & & & & & & & $x$ & & $x$ & & & $x$ & & & \\
\hline DG-68 & $\mathrm{x}$ & $\mathrm{x}$ & & & $\mathrm{x}$ & $\mathrm{x}$ & & & & & & & & $\mathrm{x}$ & & & & $\mathrm{x}$ & $\mathrm{x}$ & $\mathrm{x}$ & & $\mathrm{x}$ \\
\hline Dt-8 (118.0m) & $x$ & $x$ & $x$ & & $x$ & $x$ & & & & & & & & & $x$ & $x$ & & & $x$ & & & \\
\hline Dt-8 (129.5m) & $\mathrm{x}$ & $\mathrm{x}$ & $\mathrm{x}$ & & $x^{a}$ & $\mathrm{x}$ & & & & & & & $\mathrm{x}$ & $\mathrm{x}$ & $\mathrm{x}$ & & $\mathrm{x}$ & $\mathrm{x}$ & $\mathrm{x}$ & & & \\
\hline$D t-8(207.8 m)$ & $x$ & $x$ & $x$ & $x$ & $x$ & $x$ & & & & & & & & $x$ & & $x$ & & $x$ & $x$ & & & \\
\hline
\end{tabular}

Abbreviations of mineral names after Bucher \& Frey (1994) except Bafs = Ba-feldspar, $\mathrm{Chl} / \mathrm{Sm}=$ chlorite/smectite mixed-layered mineral. Meta-igneous rocks are indicated in roman, metasedimentary rocks in italic. ${ }^{\text {a }}$ Celadonite in traces. 
Table 3. Bulk rock major element chemical compositions (wt \%).

\begin{tabular}{|c|c|c|c|c|c|c|c|c|c|c|c|}
\hline \multirow[b]{2}{*}{ Sample } & \multicolumn{6}{|c|}{ Metabasites } & \multicolumn{5}{|c|}{ Metasediments } \\
\hline & DG-56 & DG-33 & DG-16 & DG-82 & DG-68 & Dt-8 (129.5m) & DG-62 & DG-20 & DG-73 & Dt-8 (118.0m) & Dt-8 (207.8m) \\
\hline $\mathrm{SiO}_{2}$ & 48.27 & 47.77 & 48.49 & 46.37 & 58.93 & 33.78 & 68.61 & 54.33 & 52.34 & 53.16 & 75.00 \\
\hline $\mathrm{TiO}_{2}$ & 1.96 & 1.55 & 1.69 & 1.44 & 1.35 & 3.64 & 0.67 & 0.83 & 0.57 & 0.99 & 0.44 \\
\hline $\mathrm{Al}_{2} \mathrm{O}_{3}$ & 13.99 & 14.56 & 14.72 & 17.08 & 17.40 & 12.78 & 13.88 & 20.56 & 17.44 & 19.72 & 8.60 \\
\hline $\mathrm{Fe}_{2} \mathrm{O}_{3}$ & 2.84 & 2.82 & 4.46 & 3.25 & 3.48 & 0.36 & 1.24 & 2.10 & 1.79 & 0.57 & 0.31 \\
\hline $\mathrm{FeO}$ & 7.00 & 8.09 & 6.38 & 5.72 & 3.08 & 10.11 & 3.24 & 6.18 & 2.79 & 8.38 & 2.02 \\
\hline $\mathrm{MnO}$ & 0.13 & 0.18 & 0.20 & 0.14 & 0.07 & 0.26 & 0.23 & 0.43 & 0.03 & 0.12 & 0.06 \\
\hline $\mathrm{MgO}$ & 5.88 & 6.73 & 6.93 & 7.60 & 1.17 & 3.85 & 2.90 & 1.95 & 2.88 & 3.19 & 1.60 \\
\hline $\mathrm{CaO}$ & 8.64 & 9.95 & 7.90 & 4.85 & 2.72 & 11.80 & 0.83 & 1.58 & 6.44 & 1.20 & 4.00 \\
\hline $\mathrm{Na}_{2} \mathrm{O}$ & 4.67 & 3.80 & 4.75 & 3.75 & 4.55 & 4.42 & 0.83 & 1.03 & 0.73 & 0.77 & 3.95 \\
\hline $\mathrm{K}_{2} \mathrm{O}$ & 0.19 & 0.13 & 0.10 & 2.43 & 2.78 & 0.80 & 2.56 & 3.44 & 5.41 & 4.27 & 0.42 \\
\hline${ }^{-} \mathrm{H}_{2} \mathrm{O}$ & 0.32 & 0.15 & 0.22 & 0.22 & 0.09 & 0.10 & 0.42 & 0.80 & 0.34 & 0.15 & 0.06 \\
\hline${ }^{+} \mathrm{H}_{2} \mathrm{O}$ & 3.59 & 3.68 & 3.58 & 4.62 & 2.47 & 2.14 & 4.11 & 5.63 & 4.51 & 5.35 & 0.65 \\
\hline $\mathrm{CO}_{2}$ & 2.32 & 0.27 & 0.07 & 1.62 & 1.38 & 14.19 & 0.24 & 0.78 & 4.22 & 1.26 & 3.11 \\
\hline $\mathrm{P}_{2} \mathrm{O}_{5}$ & 0.27 & 0.14 & 0.16 & 0.25 & 0.56 & 0.81 & 0.08 & 0.12 & 0.12 & 0.27 & 0.04 \\
\hline Total & 100.10 & 99.82 & 100.10 & 99.34 & 100.03 & 99.04 & 99.84 & 99.76 & 99.61 & 99.40 & 100.26 \\
\hline
\end{tabular}

Table 4. Corrected chlorite and illite crystallinity values measured on $<2 \mu \mathrm{m}$ fraction, sedimented mounts (values in $\Delta^{\circ} 2 \Theta, \mathrm{Cu}_{\alpha}$ ).

\begin{tabular}{|c|c|c|c|c|c|c|c|c|c|c|c|c|}
\hline \multirow[b]{2}{*}{ Sample } & \multicolumn{4}{|c|}{$[\mathrm{ChC}(001)]$ c. $14 \AA$ reflection } & \multicolumn{4}{|c|}{$[\mathrm{ChC}(002)]$ c. $7 \AA$ reflection } & \multicolumn{4}{|c|}{$\mathrm{IC}(002)$ c. $10 \AA$ reflection } \\
\hline & $\mathrm{AD}$ & EG & $\mathrm{Mg}, \mathrm{AD}$ & $\mathrm{Mg}, \mathrm{EG}$ & $\mathrm{AD}$ & EG & $\mathrm{Mg}, \mathrm{AD}$ & $\mathrm{Mg}, \mathrm{EG}$ & $\mathrm{AD}$ & EG & $\mathrm{Mg}, \mathrm{AD}$ & $\mathrm{Mg}, \mathrm{ED}$ \\
\hline DG-56 & 0.418 & 0.453 & 0.416 & 0.444 & 0.401 & 0.422 & 0.387 & 0.416 & & & & \\
\hline$D G-62$ & 0.391 & 0.410 & 0.370 & 0.402 & 0.377 & 0.368 & 0.361 & 0.374 & 0.698 & 0.565 & 0.743 & 0.580 \\
\hline DG-33 & 0.495 & 0.629 & 0.462 & 0.720 & 0.449 & 0.534 & 0.407 & 0.511 & & & & \\
\hline$D G-20$ & 0.467 & 0.427 & 0.448 & 0.371 & 0.382 & 0.371 & 0.362 & 0.358 & 0.606 & 0.530 & 0.622 & 0.504 \\
\hline DG-16 & 0.455 & 0.491 & 0.438 & 0.419 & 0.439 & 0.455 & 0.399 & 0.421 & & & & \\
\hline DG-82 & 0.329 & 0.323 & 0.278 & 0.313 & 0.301 & 0.309 & 0.281 & 0.291 & 0.331 & 0.303 & 0.285 & 0.308 \\
\hline$D G-73$ & 0.311 & 0.346 & 0.373 & 0.269 & 0.288 & 0.306 & 0.267 & 0.269 & 0.271 & 0.269 & 0.265 & 0.254 \\
\hline DG-68 & 0.344 & 0.324 & 0.333 & 0.338 & 0.326 & 0.307 & 0.298 & 0.298 & 0.311 & 0.268 & 0.275 & 0.271 \\
\hline$D t-8(118.0 m)$ & 0.315 & 0.285 & 0.331 & 0.305 & 0.287 & 0.267 & 0.288 & 0.281 & 0.307 & 0.293 & 0.318 & 0.304 \\
\hline Dt-8 (129.5m) & 0.295 & 0.296 & n.m. & n.m. & 0.278 & 0.289 & n.m. & n.m. & $0.443^{\mathrm{a}}$ & $0.419^{\mathrm{a}}$ & n.m. & n.m. \\
\hline Dt-8 (207.8m) & 0.288 & 0.273 & 0.388 & 0.266 & 0.264 & 0.266 & 0.316 & 0.263 & & & & \\
\hline
\end{tabular}

FWHM measured on $\mathrm{AD}=$ air-dried, $\mathrm{EG}=$ ethylene glycol-solvated, $\mathrm{Mg}, \mathrm{AD}=\mathrm{MgCl}_{2}$-saturated and air-dried, $\mathrm{Mg}, \mathrm{EG}=\mathrm{MgCl}_{2}$-saturated and ethylene glycolated mounts. $\mathrm{Metasediments}$ are indicated in italics. n.m., not measured. ${ }^{\text {a }}$ Celadonite-glauconite.

and $\mathrm{ChC}(002)$. $\mathrm{Mg}$ saturation caused minor broadening of the first two basal reflections in the lowest grade shale and slate (DG-62, DG-20) samples and metabasite samples DG-33, DG-16 and DG-82. In contrast, $\mathrm{Mg}$ saturation caused considerable peak broadening for the $14 \AA$ reflection of slate sample DG-73, and for the 14 and $7 \AA$ reflections of the metasandstone sample Dt-8 (207.8m), but did not cause a change in the FWHM values of the chlorite peaks of metabasite samples DG-56 and DG-68, and slate sample Dt-8 (118.0m).

In general, EG solvation had a greater effect on chlorite FWHM values than did $\mathrm{Mg}$ saturation. The absolute values of differences in FWHM data show a decreasing trend with increasing grade. Considering the $14 \AA$ reflection, glycolation caused broadening (samples DG-56, DG-62, DG-33, DG-16, DG-73) or sharpening (samples DG-20, Dt-8 (118.0m)), but practically no changes were observed for samples DG-82, DG-68, Dt-8 (129.5m) and Dt-8 (207.8m). With respect to the $7 \AA$ reflection, however, glycolation caused negligible changes (mostly slight broadening) which were within the error of FWHM measurements, except for the considerable broadening observed for metabasite sample DG-33.

\section{Apparent mean crystallite size and lattice strain calculated from XRD line profiles}

Table 5 contains the apparent mean crystallite size values of illite-muscovite and chlorite determined by the Scherrer equation, and Tables 6,7 and 8 the mean crystallite size and lattice strain values calculated by

Table 5. Apparent mean crystallite size values of chlorite and illite-muscovite in A, calculated from the FWHM data of XRD basal reflections ${ }^{\mathrm{a}}$ by the Scherrer equation ${ }^{\mathrm{b}}$. Air-dried (AD),

$<2 \mu \mathrm{m}$ fraction samples.

\begin{tabular}{lccc}
\hline $\begin{array}{l}\text { Sample } \\
\text { Reflection }\end{array}$ & $\begin{array}{c}\text { Chlorite } \\
14 \AA\end{array}$ & $\begin{array}{c}\text { Chlorite } \\
7 \AA\end{array}$ & $\begin{array}{c}\text { Illite-muscovite } \\
10 \AA\end{array}$ \\
\hline DG-56 & 300 & 341 & 132 \\
$D G-62$ & 326 & 392 & 161 \\
DG-33 & 216 & 273 & 516 \\
$D G-20$ & 236 & 378 & 1052 \\
DG-16 & 245 & 286 & 630 \\
DG-82 & 484 & 731 & 660 \\
$D G-73$ & 561 & 867 & \\
DG-68 & 434 & 570 & \\
$D t-8(118.0 m)$ & 543 & 878 & 1004 \\
Dt-8 $(129.5 \mathrm{~m})$ & 644 & 1292 & \\
$D t-8(207.8 m)$ & 688 & & \\
\hline
\end{tabular}

${ }^{a}$ For instrumental correction, natural 'standards' of chlorite (CHL-1) and muscovite (SL-8) were applied (see Árkai et al., 1996). ${ }^{\text {b }}$ For details of calculation, see Merriman et al. (1990). 
Table 6. Apparent mean crystallite size and lattice strain values of illite-muscovite calculated from the $10 \AA$ reflection by the Voigt method, using single-line Fourier analysis (standard for instrumental correction: SL-8, $10 \AA$ A).

\begin{tabular}{|c|c|c|c|c|c|c|c|c|c|c|c|c|}
\hline \multirow[b]{2}{*}{ Sample } & \multicolumn{4}{|c|}{ Mean crystallite size $(\AA)$} & \multicolumn{4}{|c|}{ Mean lattice strain (\%) } & \multicolumn{4}{|c|}{ Error of fitting } \\
\hline & $\mathrm{AD}$ & EG & $\mathrm{Mg}, \mathrm{AD}$ & $\mathrm{Mg}, \mathrm{EG}$ & $\mathrm{AD}$ & EG & $\mathrm{Mg}, \mathrm{AD}$ & $\mathrm{Mg}, \mathrm{EG}$ & $\mathrm{AD}$ & EG & $\mathrm{Mg}, \mathrm{AD}$ & $\mathrm{Mg}, \mathrm{EG}$ \\
\hline$D G-62$ & 156 & 201 & 188 & 204 & 1.35 & 1.24 & 1.49 & 1.23 & 0.039 & 0.024 & 0.025 & 0.048 \\
\hline DG-20 & 210 & 207 & 206 & 236 & 1.16 & 1.26 & 1.33 & 1.09 & 0.019 & 0.038 & 0.023 & 0.026 \\
\hline DG-82 & 693 & 724 & 757 & 795 & 0.45 & 0.46 & 0.53 & 0.36 & 0.029 & 0.034 & 0.039 & 0.027 \\
\hline$D G-73$ & 879 & 852 & 1054 & 1113 & 0.54 & 0.54 & 0.49 & 0.49 & 0.011 & 0.009 & 0.009 & 0.007 \\
\hline DG-68 & 711 & 729 & 690 & 789 & 0.55 & 0.66 & 0.70 & 0.58 & 0.010 & 0.016 & 0.010 & 0.011 \\
\hline$D t-8(118.0 m)$ & 625 & 678 & 599 & 613 & 0.64 & 0.64 & 0.62 & 0.67 & 0.006 & 0.006 & 0.010 & 0.006 \\
\hline
\end{tabular}

$\mathrm{AD}=$ air-dried, $\mathrm{EG}=$ ethylene glycol-solvated, $\mathrm{Mg}, \mathrm{AD}=\mathrm{MgCl}_{2}$-saturated and air-dried, $\mathrm{Mg}, \mathrm{EG}=\mathrm{MgCl}_{2}$-saturated and glycolated mounts; metasediments are indicated in italics.

Table 7. Apparent mean crystallite size and lattice strain values of chlorite calculated from the $14 \AA$ reflection by the Voigt method, using single-line Fourier analysis (standard for instrumental correction: CHL-1, 14 A).

\begin{tabular}{|c|c|c|c|c|c|c|c|c|c|c|c|c|}
\hline \multirow[b]{2}{*}{ Sample } & \multicolumn{4}{|c|}{ Mean crystallite size $(\AA)$} & \multicolumn{4}{|c|}{ Mean lattice strain (\%) } & \multicolumn{4}{|c|}{ Error of fitting } \\
\hline & $\mathrm{AD}$ & EG & $\mathrm{Mg}, \mathrm{AD}$ & $\mathrm{Mg}, \mathrm{EG}$ & $\mathrm{AD}$ & EG & $\mathrm{Mg}, \mathrm{AD}$ & $\mathrm{Mg}, \mathrm{EG}$ & $\mathrm{AD}$ & EG & $\mathrm{Mg}, \mathrm{AD}$ & $\mathrm{Mg}, \mathrm{EG}$ \\
\hline DG-56 & 339 & 292 & 366 & 372 & 1.04 & 1.32 & 0.98 & 0.67 & 0.029 & 0.023 & 0.042 & 0.019 \\
\hline$D G-62$ & 314 & 260 & 327 & 377 & 1.22 & 1.66 & 1.27 & 0.49 & 0.026 & 0.050 & 0.040 & 0.069 \\
\hline DG-33 & 231 & 220 & 250 & 203 & 1.38 & 0.89 & 1.52 & 1.69 & 0.033 & 0.224 & 0.057 & 0.194 \\
\hline$D G-20$ & 227 & 147 & 200 & 490 & 1.97 & 2.92 & 1.63 & 0.42 & 0.177 & 0.204 & 0.579 & 0.086 \\
\hline DG-16 & 323 & 300 & 359 & 413 & 0.76 & 0.62 & 0.82 & 0.37 & 0.055 & 0.103 & 0.037 & 0.041 \\
\hline DG-82 & 518 & 684 & 671 & 667 & 0.89 & 0.41 & 0.96 & 0.82 & 0.014 & 0.159 & 0.014 & 0.020 \\
\hline$D G-73$ & 397 & 458 & 377 & 1168 & 1.55 & 1.30 & 1.62 & 0.07 & 0.045 & 0.046 & 0.053 & 0.058 \\
\hline DG-68 & 365 & 640 & 319 & 452 & 1.56 & 0.73 & 1.86 & 1.40 & 0.125 & 0.123 & 0.040 & 0.069 \\
\hline Dt-8 (118.0m) & 503 & 731 & 434 & 735 & 1.11 & 0.67 & 1.20 & 0.49 & 0.021 & 0.022 & 0.049 & 0.033 \\
\hline Dt-8 (129.5m) & 694 & 823 & n.c. & n.c. & 0.97 & 0.80 & n.c. & n.c. & 0.085 & 0.106 & n.c. & n.c. \\
\hline$D t-8(207.8 m)$ & 661 & 691 & 570 & 1032 & 1.00 & 0.93 & 0.27 & 0.57 & 0.019 & 0.036 & 0.063 & 0.045 \\
\hline
\end{tabular}

$\mathrm{AD}=$ air-dried, $\mathrm{EG}=$ ethylene glycol-solvated, $\mathrm{Mg}, \mathrm{AD}=\mathrm{MgCl}_{2}$-saturated and air-dried, $\mathrm{Mg}, \mathrm{EG}=\mathrm{MgCl}_{2}$-saturated and glycolated mounts; metasediments are indicated in italics; n.c., not calculated.

Table 8. Apparent mean crystallite size and lattice strain values of chlorite calculated from the $7 \AA$ reflection by the Voigt method, using single-line Fourier analysis (standard for instrumental correction: CHL-1, 7 Å).

\begin{tabular}{|c|c|c|c|c|c|c|c|c|c|c|c|c|}
\hline \multirow[b]{2}{*}{ Sample } & \multicolumn{4}{|c|}{ Mean crystallite size $(\AA)$} & \multicolumn{4}{|c|}{ Mean lattice strain (\%) } & \multicolumn{4}{|c|}{ Error of fitting } \\
\hline & $\mathrm{AD}$ & EG & $\mathrm{Mg}, \mathrm{AD}$ & $\mathrm{Mg}, \mathrm{EG}$ & $\mathrm{AD}$ & EG & $\mathrm{Mg}, \mathrm{AD}$ & $\mathrm{Mg}, \mathrm{EG}$ & $\mathrm{AD}$ & EG & $\mathrm{Mg}, \mathrm{AD}$ & $\mathrm{Mg}, \mathrm{EG}$ \\
\hline DG-56 & 433 & 407 & 518 & 426 & 0.35 & 0.22 & 0.13 & 0.35 & 0.021 & 0.016 & 0.007 & 0.009 \\
\hline$D G-62$ & 439 & 437 & 462 & 464 & 0.44 & 0.45 & 0.44 & 0.38 & 0.009 & 0.008 & 0.004 & 0.007 \\
\hline DG-33 & 310 & 246 & 393 & 226 & 0.53 & 0.67 & 0.45 & 0.88 & 0.067 & 0.017 & 0.007 & 0.017 \\
\hline$D G-20$ & 574 & 466 & 526 & 555 & 0.29 & 0.42 & 0.33 & 0.32 & 0.004 & 0.005 & 0.006 & 0.003 \\
\hline DG-16 & 440 & 320 & 460 & 416 & 0.32 & 0.07 & 0.23 & 0.19 & 0.041 & 0.079 & 0.006 & 0.007 \\
\hline DG-82 & 745 & 677 & 971 & 821 & 0.34 & 0.41 & 0.33 & 0.37 & 0.011 & 0.007 & 0.004 & 0.007 \\
\hline$D G-73$ & 728 & 668 & 1184 & 1025 & 0.42 & 0.38 & 0.21 & 0.36 & 0.006 & 0.008 & 0.009 & 0.009 \\
\hline DG-68 & 607 & 727 & 689 & 735 & 0.46 & 0.44 & 0.45 & 0.41 & 0.009 & 0.013 & 0.007 & 0.112 \\
\hline Dt-8 (118.0m) & 992 & 914 & 874 & 854 & 0.21 & 0.36 & 0.30 & 0.38 & 0.004 & 0.004 & 0.004 & 0.006 \\
\hline Dt-8 (129.5m) & 925 & 775 & n.c. & n.c. & 0.29 & 0.41 & n.c. & n.c. & 0.038 & 0.010 & n.c. & n.c. \\
\hline$D t-8(207.8 m)$ & 907 & 812 & 859 & 1178 & 0.39 & 0.46 & 0.29 & 0.25 & 0.008 & 0.015 & 0.006 & 0.007 \\
\hline
\end{tabular}

$\mathrm{AD}=$ air-dried, $\mathrm{EG}=$ ethylene glycol-solvated, $\mathrm{Mg}, \mathrm{AD}=\mathrm{MgCl}_{2}$-saturated and air-dried, $\mathrm{Mg}, \mathrm{EG}=\mathrm{MgCl}_{2}$-saturated and glycolated mounts; metasediments are indicated in italics; n.c., not calculated.

the Voigt method. Table 9 summarizes the mean size values obtained by Krumm's WINFIT $2.1^{\circledR}$ program.

A surprisingly good fit is found between the Scherrer and Voigt size results. With increasing metamorphic grade (i.e. with decreasing crystallinity values), the apparent mean crystallite sizes increase, the range of changes being larger for the Scherrer results than for the Voigt data; the ranges are larger for illite-muscovite than for chlorite in the cases of both methods. In contrast to illite-muscovite, for which the lattice strain values of diagenetic samples are significantly larger than those of the higher grade samples, no systematic changes were found in chlorite lattice strain values as a function of metamorphic grade.

The mean crystallite size data of chlorite, calculated using the WINFIT $2.1{ }^{\circledR}$ single-line Fourier technique, are significantly smaller than those obtained by the Scherrer and Voigt methods. The trends as a function of metamorphic grade (crystallinity) can only be detected in those datasets for which the slopes of the linear fits to the distributions of the Fourier coefficients are used (Table 9). The mean size data calculated by the Warren-Averbach method are apparently smaller than those of the single-line Fourier method, and 
Table 9. Mean crystallite size values of chlorite in $\AA$, calculated by the WINFIT $2.1^{\circledR}$ program of Krumm (1994) from the XRD basal reflections obtained on air-dried (AD) $<2 \mu \mathrm{m}$ grain size mounts.

\begin{tabular}{|c|c|c|c|c|c|c|}
\hline \multirow[b]{3}{*}{ Sample } & \multicolumn{4}{|c|}{ Single-line Fourier analysis } & \multirow{2}{*}{\multicolumn{2}{|c|}{$\begin{array}{c}\text { Warren-Averbach } \\
\text { analysis (14 and } 7 \AA \text { ) }\end{array}$}} \\
\hline & \multicolumn{2}{|c|}{$14 \AA$} & \multicolumn{2}{|c|}{$7 \AA$} & & \\
\hline & Distr. & Slope & Distr. & Slope & Distr. & Slope \\
\hline DG-56 & 169 & 171 & 147 & 175 & 153 & 137 \\
\hline$D G-62$ & 194 & 168 & 145 & 184 & 187 & 238 \\
\hline DG-33 & 172 & 96 & 153 & 136 & 164 & 88 \\
\hline$D G-20$ & 111 & 70 & 110 & 172 & 154 & 140 \\
\hline DG-16 & 169 & 114 & 150 & 155 & 202 & 120 \\
\hline DG-82 & 152 & 379 & 138 & 664 & 150 & 333 \\
\hline$D G-73$ & 127 & 273 & 187 & 1257 & 119 & 220 \\
\hline DG-68 & 198 & 324 & 123 & 485 & 187 & 238 \\
\hline$D t-8(118.0 m)$ & 214 & 341 & 268 & 878 & 209 & 276 \\
\hline Dt-8 (129.5m) & 127 & 230 & 242 & 540 & 123 & 195 \\
\hline Dt-8 (207.8m) & 139 & 201 & 198 & 559 & 140 & 168 \\
\hline
\end{tabular}

For instrumental correction, the natural 'standard' No. CHL-1 was used (see Árkai et al., 1996).

do not show any systematic changes as a function of grade.

\section{TEM observations}

According to TEM observations and as consistent with XRD data, chlorite is the principal trioctahedral phyllosilicate at all grades, coexisting with illitemuscovite or illite/smectite in metapelites. In chlorite of low-grade metabasites (Darnó Hill and Szarvaskö complexes), there is significant mixed layering, with up to c. $25 \%$ expandable, Mg-rich, smectite- or vermiculite-like layers. Mixed layering is generally random, but corrensitic packets also occur locally. By contrast, smectite-like mixed layers are absent in chlorite of metapelites. Interstratifications of berthierine and chlorite occur in specimens only from diagenetic pelites. The Fe-rich berthierine layers occur randomly or as small packets of layers within large packets that are dominated by chlorite layers, or as separate, large crystallites up to $500 \AA$ thick.

With increasing metamorphic grade, the proportions of mixed layering decrease both in illite-muscovite and chlorite. In epizonal samples of borehole Dt-8 and the Bükk Mountains, relic authigenic mixed layering is absent, but minor berthierine, smectite and illite/ smectite occur at the rims of larger crystals of chlorite or muscovite, both in metabasites and metapelites. These features are inferred to be due to retrograde overprinting. Chlorite crystals of higher grades display similar rimming in metapelites and metabasites. Chlorite crystals in a pair of associated metapelites and metabasites of epizone-greenschist grade are large (c. $1000 \AA$ thick) and defects are rare or absent, stacking faults being observed only rarely.

Figure 3 displays histograms of chlorite crystallite size data measured parallel to $\mathrm{c}^{*}$, directly on TEM images. All of the investigated rock samples of the two lithologies display size distributions characterized by positive skewness; the medians and especially the modes are therefore all smaller than the mean values. The ranges of the size values become broader and the size distributions become more and more irregular with increasing metamorphic grade. In spite of the relatively small numbers of size measurements $(n$, the number of measurements, varies between 33 and 120 for different samples), fairly good agreement is found between the statistical parameters calculated for the metapelite-metabasite rock pairs, to a first approximation.

\section{Composition of chlorite}

Average chemical compositions and their standard deviations determined by AEM and EMP are given in Tables 10 and 11, respectively. All normalized compositions correspond to a trioctahedral structure (tri-tri). The $\mathrm{Mg}$ : Fe ratio varies considerably, however, some chlorite having $\mathrm{Fe}>\mathrm{Mg}$ and therefore being classed as chamosite, whereas others have $\mathrm{Mg}>\mathrm{Fe}$ and are therefore classed as clinochlore. High values of the $\mathrm{Mg}: \mathrm{Fe}$ ratio of chlorite are, to a first approximation, correlated with relatively high values of the $\mathrm{Mg}$ : Fe ratio of the corresponding rock (Table 3), but there are exceptions to this rule. As for the bulk rock analyses, there is no clearly defined correspondence between rock type (metabasite vs. metapelite) and $\mathrm{Mg}:$ Fe ratio. All formulae in Tables 10 and 11 are normalized both to an ideal trioctahedral formula with a total of 20 octahedral + tetrahedral cations, and to a charge-balanced formula with 28 oxygen atoms. In addition, all iron was assumed to be ferrous iron. The formulae based on normalization to 20 cations, especially for low-grade samples, are not chargebalanced, having $\mathrm{Al}^{\mathrm{VI}}>\mathrm{Al}^{\mathrm{IV}}$. This, especially in light of the presence of $\mathrm{Ca}, \mathrm{Na}$ and $\mathrm{K}$ in analyses, implies that analyses were in part contaminated by inclusion of materials other than ideal chlorite layers within the volume of interaction of the electron beam and sample. These factors are discussed below.

\section{DISCUSSION}

\section{Comparison of $\mathrm{ChC}$ values for metabasites and metapelites}

The principal aim of this research was to compare the extent of reaction progress in trioctahedral phyllosilicates in coeval pairs of metabasites and metapelites. These pairs are very different in modal composition (Table 2), bulk rock composition (Table 3), textures and mineral assemblages. Table 4 shows, however, that ChC values for metabasite-metapelite pairs are nearly identical, within error. The $\mathrm{ChC}$ values for metabasites are all slightly greater than the values for corresponding pelites, but only by $c$. 0.02 for most pairs.

This correlation is especially surprising if the values of $\mathrm{ChC}$ are functions of non-equilibrium states of the trioctahedral minerals (Essene \& Peacor, 1995; 
Metabasic rocks
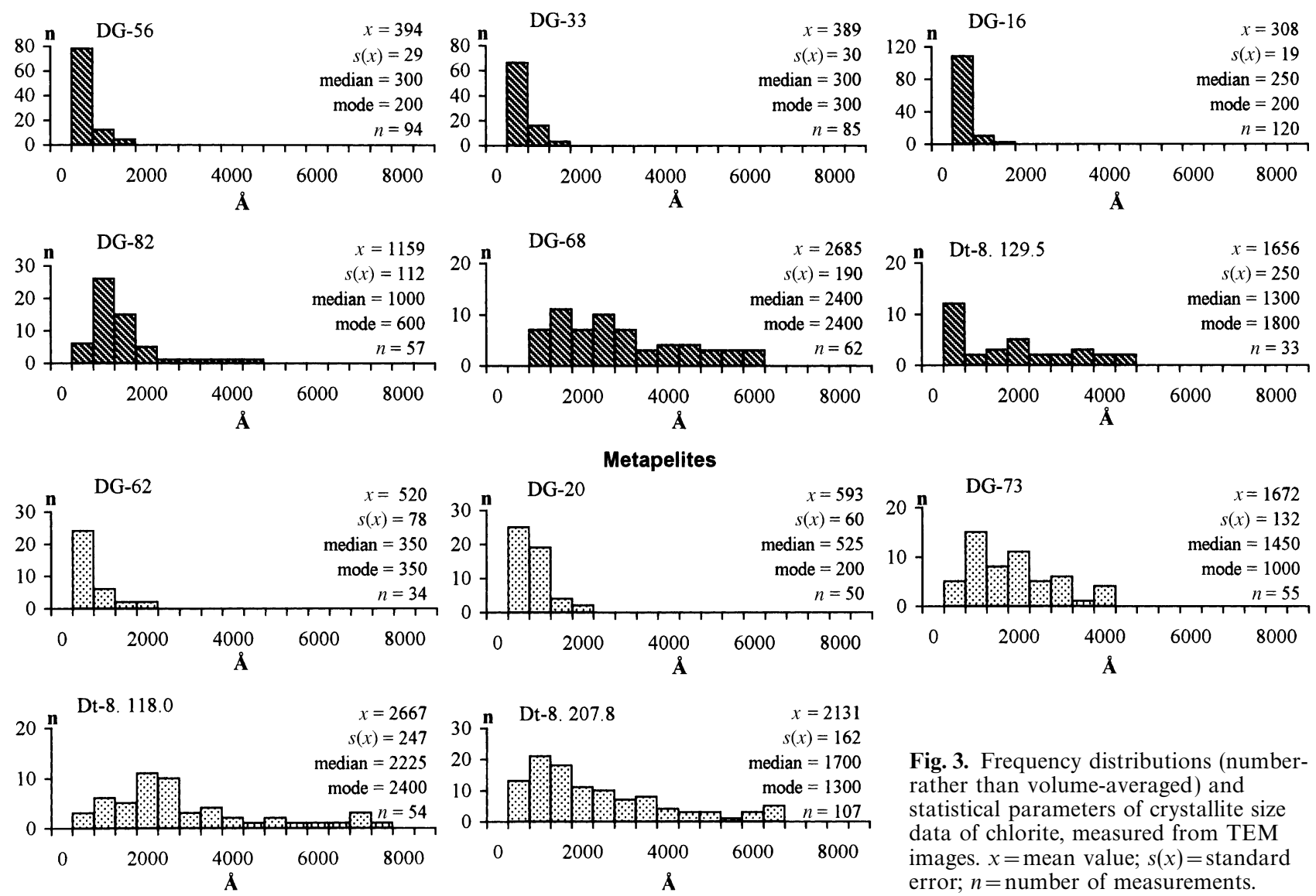

Fig. 3. Frequency distributions (numberrather than volume-averaged) and statistical parameters of crystallite size data of chlorite, measured from TEM images. $x=$ mean value; $s(x)=$ standard error; $n=$ number of measurements.

Merriman \& Peacor, 1999). A principal cause of changes in $\mathrm{ChC}$ is changes in crystal size distributions (see below), the smaller crystallite sizes at lower grades clearly representing a non-equilibrium state, whereas the larger crystallite sizes attained at epizone (greenschist) grades represent an approach to textural equilibrium. More importantly, a major cause of increasing peak breadth of XRD $00 l$ reflections in samples from lower grades is increasing proportions of mixed layering, an even more well-defined example of a metastable equilibrium state. However, the kind of mixed layering in metabasites is that of intercalation of expandable saponite- or vermiculite-like units which locally form corrensite layers, whereas $7 \AA$ berthierinelike layers occur in chlorite packets in metapelites (see below for a detailed discussion). Each is a different non-equilibrium state contributing differently to peak breadths in XRD patterns, yet the resultant peak breadths are equivalent. For example, intercalation of $7 \AA$ berthierine-like layers in chlorite packets causes preferential broadening of odd orders (e.g. 001) of $00 l$ reflections, relative to even orders (e.g. 002). Nevertheless, the values of both $\mathrm{ChC}(001)$ and $\mathrm{ChC}(002)$ for coeval pairs are generally very similar. The differences in mixed layering components provide a possible reason for the slightly larger $\mathrm{ChC}$ values for metabasites, but this seems unlikely as the differences also occur for high-grade samples where mixed layering is absent or nearly so.

\section{ChC vs. metamorphic grade}

The degree of reaction progress for coeval metabasites and metapelites is therefore approximately equivalent, even though each is based on a non-equivalent state, for rock pairs which are optimally different in composition and texture. It is therefore tempting to equate reaction progress directly with metamorphic grade (temperature), at least to a first approximation. Not only do the crystallinity data for pairs of metapelites and metabasites show similar trends with increasing grade, but there is a parallel trend in IC values for those samples where IC could be measured (Table 4), differences in IC being well documented as a measure of reaction progress and of relative grade of formation. That is, the XRD crystallinity data presented here confirm the following conclusions of Árkai (1991), Árkai et al. (1995b), Warr (1996) and Árkai \& Sadek Ghabrial (1997).

1 The ChC method is a useful tool that is complemen- 
Table 10. Average chemical compositions of chlorite determined by AEM.

\begin{tabular}{|c|c|c|c|c|c|c|c|c|c|c|c|c|c|c|c|c|c|c|}
\hline \multirow[t]{2}{*}{$\begin{array}{l}\text { Sample } \\
n\end{array}$} & \multicolumn{3}{|c|}{$\begin{array}{c}\text { DG-56 } \\
17\end{array}$} & \multicolumn{3}{|c|}{$\begin{array}{c}\text { DG-62 } \\
5\end{array}$} & \multicolumn{3}{|c|}{$\begin{array}{c}\text { DG-33 } \\
12\end{array}$} & \multicolumn{3}{|c|}{$\begin{array}{c}\text { DG-20 } \\
7\end{array}$} & \multicolumn{3}{|c|}{$\begin{array}{c}\text { DG-16 } \\
7\end{array}$} & \multicolumn{3}{|c|}{$\begin{array}{c}\text { DG-82 } \\
12\end{array}$} \\
\hline & $x^{\mathrm{a}}$ & $s$ & $x^{\mathrm{b}}$ & $x^{\mathrm{a}}$ & $s$ & $x^{\mathrm{b}}$ & $x^{\mathrm{a}}$ & $s$ & $x^{\mathrm{b}}$ & $x^{\mathrm{a}}$ & $s$ & $x^{\mathrm{b}}$ & $x^{\mathrm{a}}$ & $s$ & $x^{\mathrm{b}}$ & $x^{\mathrm{a}}$ & s & $x^{\mathrm{b}}$ \\
\hline $\mathrm{Si}$ & 6.29 & 0.25 & 6.17 & 6.30 & 0.76 & 5.99 & 6.01 & 0.41 & 5.89 & 6.04 & 1.05 & 5.80 & 5.98 & 0.38 & 5.90 & 5.74 & 0.24 & 5.69 \\
\hline $\mathrm{Al}^{\mathrm{IV}}$ & 1.71 & 0.25 & 1.84 & 1.70 & 0.76 & 2.01 & 1.99 & 0.41 & 2.11 & 1.96 & 1.05 & 2.20 & 2.02 & 0.38 & 2.10 & 2.26 & 0.24 & 2.31 \\
\hline $\mathrm{Al}^{\mathrm{t}}$ & 3.88 & 0.23 & 3.80 & 5.38 & 0.68 & 5.12 & 4.16 & 0.48 & 4.08 & 5.73 & 0.49 & 5.50 & 4.23 & 0.46 & 4.17 & 4.50 & 0.23 & 4.46 \\
\hline $\mathrm{Al}^{\mathrm{VI}}$ & 2.17 & 0.33 & 1.96 & 3.68 & 1.31 & 3.11 & 2.17 & 0.68 & 1.97 & 3.77 & 1.07 & 3.30 & 2.21 & 0.31 & 2.07 & 2.24 & 0.40 & 2.15 \\
\hline $\mathrm{Ti}$ & 0.02 & 0.06 & 0.02 & 0.03 & 0.07 & 0.03 & 0.04 & 0.09 & 0.04 & 0.00 & 0.00 & 0.00 & 0.03 & 0.07 & 0.03 & 0.00 & 0.00 & 0.00 \\
\hline $\mathrm{Fe}^{2+\mathrm{c}}$ & 4.27 & 0.24 & 4.18 & 3.31 & 1.05 & 3.15 & 6.25 & 1.26 & 6.13 & 5.27 & 0.95 & 5.06 & 4.78 & 0.25 & 4.71 & 3.49 & 0.42 & 3.46 \\
\hline $\mathrm{Mg}$ & 5.33 & 0.19 & 5.22 & 4.48 & 0.32 & 4.26 & 3.20 & 0.86 & 3.14 & 2.43 & 1.07 & 2.33 & 4.65 & 0.38 & 4.59 & 6.10 & 0.21 & 6.05 \\
\hline $\mathrm{Mn}$ & 0.18 & 0.06 & 0.18 & 0.43 & 0.15 & 0.41 & 0.29 & 0.13 & 0.28 & 0.45 & 0.14 & 0.43 & 0.25 & 0.05 & 0.25 & 0.17 & 0.05 & 0.17 \\
\hline $\mathrm{Cr}$ & 0.03 & 0.07 & 0.03 & 0.07 & 0.10 & 0.07 & 0.05 & 0.12 & 0.05 & 0.08 & 0.08 & 0.08 & 0.08 & 0.11 & 0.08 & 0.00 & 0.01 & 0.00 \\
\hline Sum $R^{\mathrm{VI}}$ & 12.00 & & 11.59 & 12.00 & & 11.03 & 12.00 & & 11.61 & 12.00 & & 11.20 & 12.00 & & 11.73 & 12.00 & & 11.83 \\
\hline $\mathrm{Ca}$ & 0.19 & 0.12 & 0.19 & 0.16 & 0.15 & 0.15 & 0.38 & 0.09 & 0.37 & 0.08 & 0.11 & 0.08 & 0.21 & 0.16 & 0.21 & 0.10 & 0.10 & 0.10 \\
\hline $\mathrm{Na}$ & 0.13 & 0.25 & 0.13 & 0.00 & 0.00 & 0.00 & 0.00 & 0.00 & 0.00 & 0.11 & 0.28 & 0.11 & 0.00 & 0.00 & 0.00 & 0.22 & 0.24 & 0.22 \\
\hline $\mathrm{K}$ & 0.01 & 0.05 & 0.01 & 0.44 & 0.16 & 0.42 & 0.06 & 0.16 & 0.06 & 0.19 & 0.24 & 0.18 & 0.04 & 0.10 & 0.04 & 0.09 & 0.15 & 0.09 \\
\hline $\operatorname{Sum}(\mathrm{Ca}+\mathrm{Na}+\mathrm{K})$ & 0.33 & 0.27 & 0.33 & 0.60 & 0.29 & 0.57 & 0.44 & 0.20 & 0.43 & 0.37 & 0.35 & 0.37 & 0.25 & 0.22 & 0.25 & 0.41 & 0.26 & 0.55 \\
\hline Total & 20.33 & 0.27 & 19.92 & 20.60 & 0.29 & 19.60 & 20.44 & 0.20 & 20.04 & 20.37 & 0.35 & 19.57 & 20.25 & 0.22 & 19.98 & 20.41 & 0.26 & 20.38 \\
\hline$X_{\mathrm{Mg}}$ & 0.44 & 0.02 & 0.44 & 0.37 & 0.03 & 0.37 & 0.27 & 0.07 & 0.27 & 0.20 & 0.09 & 0.20 & 0.39 & 0.03 & 0.39 & 0.51 & 0.03 & 0.51 \\
\hline $\mathrm{Fe}^{2+} /\left(\mathrm{Fe}^{2+}+\mathrm{Mg}\right)$ & 0.44 & 0.02 & 0.44 & 0.42 & 0.08 & 0.43 & 0.66 & 0.09 & 0.66 & 0.69 & 0.11 & 0.69 & 0.51 & 0.03 & 0.51 & 0.36 & 0.03 & 0.36 \\
\hline $\mathrm{Si} /\left(\mathrm{Si}+\mathrm{Al}^{\mathrm{t}}\right)$ & 0.62 & 0.02 & 0.62 & 0.54 & 0.03 & 0.54 & 0.59 & 0.03 & 0.59 & 0.51 & 0.05 & 0.51 & 0.59 & 0.04 & 0.59 & 0.56 & 0.01 & 0.56 \\
\hline $\mathrm{Si}+\mathrm{Al}^{\mathrm{t}}+\mathrm{Fe}^{2+}+\mathrm{Mg}$ & 19.77 & 0.13 & 19.37 & 19.47 & 0.13 & 18.52 & 19.63 & 0.23 & 19.24 & 19.47 & 0.16 & 18.69 & 19.64 & 0.18 & 19.37 & 19.83 & 0.05 & 19.66 \\
\hline $\mathrm{Al}^{\mathrm{t}}+\mathrm{Fe}^{2+}+\mathrm{Mg}$ & 13.48 & 0.26 & 13.20 & 13.17 & 0.72 & 12.53 & 13.62 & 0.44 & 13.35 & 13.43 & 0.98 & 12.89 & 13.66 & 0.30 & 13.47 & 14.09 & 0.23 & 13.97 \\
\hline \multirow[t]{2}{*}{$\begin{array}{l}\text { Sample } \\
n\end{array}$} & \multicolumn{3}{|c|}{ DG-73 } & \multicolumn{4}{|c|}{ DG-68 } & \multicolumn{3}{|c|}{ Dt-8 (118.0m) } & \multicolumn{4}{|c|}{ Dt-8 (129.5m) } & \multicolumn{3}{|c|}{$\begin{array}{c}\text { Dt-8 (207.8m) } \\
20\end{array}$} & \\
\hline & $x^{\mathrm{a}}$ & $s$ & $x^{\mathrm{b}}$ & & $x^{\mathrm{a}}$ & $s$ & $x^{\mathrm{b}}$ & $x^{\mathrm{a}}$ & $s$ & $x^{\mathrm{b}}$ & & $x^{\mathrm{a}}$ & $s$ & $x^{\mathrm{b}}$ & $x^{\mathrm{a}}$ & $s$ & $x^{\mathrm{b}}$ & \\
\hline $\mathrm{Si}$ & 5.57 & 0.31 & 5.50 & & 5.34 & 0.31 & 5.33 & 5.41 & 0.19 & 5.34 & & 5.23 & 0.46 & 5.19 & 5.75 & 0.46 & 5.72 & \\
\hline $\mathrm{Al}^{\mathrm{IV}}$ & 2.08 & 1.21 & 2.50 & & 2.66 & 0.31 & 2.67 & 2.59 & 0.19 & 2.66 & & 2.76 & 0.46 & 2.81 & 2.25 & 0.46 & 2.28 & \\
\hline $\mathrm{Al}^{\mathrm{t}}$ & 5.18 & 0.19 & 5.12 & & 5.27 & 0.31 & 5.26 & 5.76 & 0.17 & 5.68 & & 5.28 & 0.45 & 5.24 & 4.40 & 0.28 & 4.37 & \\
\hline $\mathrm{Al}^{\mathrm{VI}}$ & 2.43 & 0.31 & 2.62 & & 2.60 & 0.22 & 2.59 & 3.17 & 0.22 & 3.02 & & 2.51 & 0.49 & 2.43 & 2.15 & 0.40 & 2.09 & \\
\hline $\mathrm{Ti}$ & 0.00 & 0.00 & 0.00 & & 0.03 & 0.08 & 0.03 & 0.00 & 0.00 & 0.00 & & 0.06 & 0.12 & 0.06 & 0.00 & 0.02 & 0.00 & \\
\hline $\mathrm{Fe}^{2+c}$ & 4.35 & 0.26 & 4.30 & & 6.03 & 0.28 & 6.01 & 5.48 & 0.16 & 5.41 & & 6.96 & 0.49 & 6.91 & 3.59 & 0.18 & 3.57 & \\
\hline $\mathrm{Mg}$ & 4.80 & 0.31 & 4.74 & & 2.98 & 0.30 & 2.97 & 3.17 & 0.27 & 3.13 & & 2.18 & 0.40 & 2.16 & 6.14 & 0.33 & 6.10 & \\
\hline $\mathrm{Mn}$ & 0.07 & 0.07 & 0.07 & & 0.27 & 0.08 & 0.27 & 0.18 & 0.05 & 0.18 & & 0.17 & 0.12 & 0.17 & 0.08 & 0.07 & 0.08 & \\
\hline $\mathrm{Cr}$ & 0.04 & 0.09 & 0.04 & & 0.08 & 0.12 & 0.08 & 0.00 & 0.00 & 0.00 & & 0.11 & 0.10 & 0.11 & 0.04 & 0.08 & 0.04 & \\
\hline Sum $\mathrm{R}^{\mathrm{VI}}$ & 12.00 & & 11.77 & & 11.99 & & 11.95 & 12.00 & & 11.74 & & 1.99 & & 11.84 & 12.00 & & 11.88 & \\
\hline $\mathrm{Ca}$ & 0.04 & 0.05 & 0.04 & & 0.00 & 0.00 & 0.00 & 0.04 & 0.08 & 0.04 & & 0.23 & 0.15 & 0.23 & 0.01 & 0.03 & 0.01 & \\
\hline $\mathrm{Na}$ & 0.08 & 0.15 & 0.08 & & 0.06 & 0.16 & 0.06 & 0.00 & 0.00 & 0.00 & & 0.00 & 0.00 & 0.00 & 0.37 & 0.33 & 0.37 & \\
\hline $\mathrm{K}$ & 0.13 & 0.11 & 0.13 & & 0.00 & 0.00 & 0.00 & 0.10 & 0.14 & 0.10 & & 0.01 & 0.05 & 0.01 & 0.01 & 0.04 & 0.01 & \\
\hline $\mathrm{Sum}(\mathrm{Ca}+\mathrm{Na}+\mathrm{K})$ & 0.26 & 0.16 & 0.25 & & 0.06 & 0.16 & 0.06 & 0.14 & 0.19 & 0.14 & & 0.24 & 0.16 & 0.24 & 0.40 & 0.32 & 0.39 & \\
\hline Total & 20.26 & 0.16 & 20.02 & & 20.06 & 0.16 & 20.01 & 20.14 & 0.19 & 19.88 & & 0.24 & 0.16 & 20.08 & 20.40 & 0.32 & 20.27 & \\
\hline$X_{\mathrm{Mg}}$ & 0.40 & 0.03 & 0.40 & & 0.25 & 0.03 & 0.25 & 0.36 & 0.02 & 0.36 & & 0.18 & 0.03 & 0.18 & 0.51 & 0.03 & 0.51 & \\
\hline $\mathrm{Fe}^{2+} /\left(\mathrm{Fe}^{2+}+\mathrm{Mg}\right)$ & 0.48 & 0.03 & 0.48 & & 0.67 & 0.02 & 0.67 & 0.63 & 0.02 & 0.63 & & 0.75 & 0.07 & 0.75 & 0.37 & 0.02 & 0.37 & \\
\hline $\mathrm{Si} /\left(\mathrm{Si}+\mathrm{Al}^{\mathrm{t}}\right)$ & 0.52 & 0.02 & 0.52 & & 0.50 & 0.03 & 0.50 & 0.48 & 0.01 & 0.48 & & 0.50 & 0.04 & 0.50 & 0.57 & 0.03 & 0.57 & \\
\hline $\mathrm{Si}+\mathrm{Al}^{\mathrm{t}}+\mathrm{Fe}^{2+}+\mathrm{Mg}$ & 19.90 & 0.15 & 19.66 & & 19.62 & 0.22 & 19.57 & 19.82 & 0.05 & 19.56 & & 9.63 & 0.24 & 19.50 & 19.88 & 0.14 & 19.76 & \\
\hline $\mathrm{Al}^{\mathrm{t}}+\mathrm{Fe}^{2+}+\mathrm{Mg}$ & 14.33 & 0.23 & 14.16 & & 14.28 & 0.47 & 14.24 & 14.41 & 0.22 & 14.22 & & 4.40 & 1.05 & 14.31 & 14.13 & 0.42 & 14.04 & \\
\hline
\end{tabular}

$x=$ mean value; $s=$ standard deviation; $n=$ number of analyses. Cation numbers calculated on the basis of fixed cation sum: tetrahedral + octahedral cations $=20^{\mathrm{a}}$ and on the basis of 28 oxygens ${ }^{\mathrm{b}} .{ }^{\mathrm{c}}$ Total Fe calculated as $\mathrm{Fe}^{2+}$.

tary to the IC method for the determination of reaction progress in diagenetic and low-grade metamorphic zones of rocks of sedimentary origin, especially in cases where application of the IC method is problematic. 2 The ChC method can also be used to determine differences in metamorphic grades of metabasites, and thus to determine indirectly the metabasite mineral facies of rocks lacking key, characteristic mineral assemblages.

Judging from the XRD characteristics of the airdried, Mg-saturated, glycolated, glycerol-solvated, $\mathrm{Mg}+\mathrm{K}$-saturated and heated, $<2 \mu \mathrm{m}$ fraction samples, the proportions of expandable mixed layers are subordinate to the dominant chlorite component, even in the diagenetic and prehnite-pumpellyite facies samples. The $10 \AA$ reflection of illite-muscovite of diagenetic samples shows significant sharpening (decrease in IC) after glycolation, indicating that there are some highly expandable, low-charge, smectitic, mixed-layered components. By contrast, glycolation causes smaller scale but systematic broadening of the chlorite $14 \AA$ and $7 \AA$ peak reflections of the low-temperature samples (Table 4). One possible contribution to this rather surprising behaviour together with the small shifts of the maxima of the $14 \AA$ peaks towards lower diffraction angles due to glycolation and glycerol treatment - concerns a possible higher interlayer charge (vermiculite-like character) of the swelling mixed layers intercalated with chlorite. A second cause is implied by the textures observed by TEM. The dioctahedral swelling component occurs primarily in illite-rich I-S packets 
Table 11. Chlorite average chemical compositions determined by EMP (wt $\%$ of oxides and cation numbers).

\begin{tabular}{|c|c|c|c|c|c|c|c|c|c|c|c|c|}
\hline \multirow[t]{2}{*}{$\begin{array}{l}\text { Sample } \\
n\end{array}$} & \multicolumn{3}{|c|}{$\begin{array}{c}\text { DG-56 } \\
9\end{array}$} & \multicolumn{3}{|c|}{$\begin{array}{c}\text { DG-33 } \\
5\end{array}$} & \multicolumn{3}{|c|}{$\begin{array}{c}\text { DG-16 } \\
5\end{array}$} & \multicolumn{3}{|c|}{$\begin{array}{c}\text { DG-82 } \\
6\end{array}$} \\
\hline & $x$ & & $s$ & $x$ & & $s$ & $x$ & & $s$ & $x$ & & $s$ \\
\hline $\mathrm{SiO}_{2}$ & 31.10 & & 0.65 & 28.18 & & 0.05 & 29.28 & & 0.60 & 28.12 & & 0.63 \\
\hline $\mathrm{TiO}_{2}$ & 0.03 & & 0.03 & 0.00 & & 0.00 & 0.08 & & 0.14 & 0.00 & & 0.00 \\
\hline $\mathrm{Al}_{2} \mathrm{O}_{3}$ & 16.15 & & 0.53 & 16.35 & & 1.12 & 18.19 & & 0.64 & 17.96 & & 0.51 \\
\hline${ }^{\circ} \mathrm{FeO}$ & 24.17 & & 0.36 & 30.95 & & 0.93 & 25.88 & & 4.06 & 19.94 & & 1.28 \\
\hline $\mathrm{MnO}$ & 0.22 & & 0.01 & 0.40 & & 0.04 & 0.39 & & 0.04 & 0.35 & & 0.03 \\
\hline $\mathrm{MgO}$ & 16.81 & & 0.41 & 10.73 & & 0.58 & 14.74 & & 3.46 & 20.72 & & 0.62 \\
\hline $\mathrm{CaO}$ & 0.50 & & 0.23 & 0.67 & & 0.11 & 0.65 & & 0.11 & 0.16 & & 0.08 \\
\hline $\mathrm{NaO}$ & 0.03 & & 0.02 & 0.03 & & 0.01 & 0.02 & & 0.01 & 0.02 & & 0.02 \\
\hline $\mathrm{K}_{2} \mathrm{O}$ & 0.04 & & 0.02 & 0.02 & & 0.01 & 0.01 & & 0.02 & 0.01 & & 0.01 \\
\hline \multirow[t]{2}{*}{ Total } & 89.05 & & 0.68 & 87.33 & & 0.89 & 89.24 & & 0.79 & 87.28 & & 1.07 \\
\hline & $x^{\mathrm{a}}$ & $x^{\mathrm{b}}$ & $s$ & $x^{\mathrm{a}}$ & $x^{\mathrm{b}}$ & $s$ & $x^{\mathrm{a}}$ & $x^{\mathrm{b}}$ & $s$ & $x^{\mathrm{a}}$ & $x^{\mathrm{b}}$ & $s$ \\
\hline $\mathrm{Si}$ & 6.50 & 6.36 & 0.13 & 6.28 & 6.15 & 0.13 & 6.35 & 6.23 & 0.13 & 5.79 & 5.78 & 0.08 \\
\hline $\mathrm{Al}^{\mathrm{IV}}$ & 1.50 & 1.64 & 0.13 & 1.72 & 1.85 & 0.13 & 1.65 & 1.77 & 0.13 & 2.21 & 2.22 & 0.08 \\
\hline $\mathrm{Al}^{\mathrm{t}}$ & 3.98 & 3.90 & & 4.30 & 4.21 & & 4.07 & 3.99 & & 4.36 & 4.35 & \\
\hline $\mathrm{Al}^{\mathrm{VI}}$ & 2.48 & 2.26 & 0.05 & 2.58 & 2.36 & 0.15 & 2.42 & 2.22 & 0.15 & 2.15 & 2.13 & 0.12 \\
\hline $\mathrm{Ti}$ & 0.01 & 0.01 & 0.01 & 0.00 & 0.00 & 0.00 & 0.01 & 0.01 & 0.01 & 0.00 & 0.00 & 0.00 \\
\hline $\mathrm{Fe}^{2+\mathrm{c}}$ & 4.23 & 4.14 & 0.08 & 5.77 & 5.65 & 0.26 & 5.10 & 5.01 & 0.10 & 3.44 & 3.43 & 0.23 \\
\hline $\mathrm{Mg}$ & 5.24 & 5.13 & 0.09 & 3.56 & 3.49 & 0.16 & 4.40 & 4.32 & 0.13 & 6.36 & 6.35 & 0.19 \\
\hline $\mathrm{Mn}$ & 0.04 & 0.04 & 0.00 & 0.08 & 0.08 & 0.01 & 0.07 & 0.07 & 0.01 & 0.06 & 0.06 & 0.01 \\
\hline Sum $R^{\mathrm{VI}}$ & 12.00 & 11.58 & & 11.99 & 11.58 & & 12.00 & 11.63 & & 12.01 & 11.97 & \\
\hline $\mathrm{Ca}$ & 0.11 & 0.11 & 0.05 & 0.16 & 0.16 & 0.03 & 0.13 & 0.13 & 0.03 & 0.04 & 0.04 & 0.02 \\
\hline $\mathrm{Na}$ & 0.01 & 0.01 & 0.01 & 0.01 & 0.01 & 0.01 & 0.01 & 0.01 & 0.00 & 0.01 & 0.01 & 0.01 \\
\hline K & 0.01 & 0.01 & 0.00 & 0.01 & 0.01 & 0.00 & 0.00 & 0.00 & 0.00 & 0.00 & 0.00 & 0.00 \\
\hline $\operatorname{Sum}(\mathrm{Ca}+\mathrm{Na}+\mathrm{K})$ & 0.13 & 0.13 & & 0.18 & 0.18 & & 0.14 & 0.14 & & 0.05 & 0.05 & \\
\hline Total & 20.13 & 19.71 & & 20.17 & 19.76 & & 20.14 & 19.77 & & 12.06 & 20.02 & \\
\hline$X_{\mathrm{Mg}}$ & 0.43 & 0.43 & & 0.29 & 0.29 & & 0.36 & 0.36 & & 0.53 & 0.53 & \\
\hline $\mathrm{Fe}^{2+} /\left(\mathrm{Fe}^{2+}+\mathrm{Mg}\right)$ & 0.45 & 0.45 & & 0.62 & 0.62 & & 0.54 & 0.54 & & 0.35 & 0.35 & \\
\hline $\mathrm{Si} /\left(\mathrm{Si}+\mathrm{Al}^{\mathrm{t}}\right)$ & 0.62 & 0.62 & & 0.59 & 0.59 & & 0.61 & 0.61 & & 0.57 & 0.57 & \\
\hline $\mathrm{Si}+\mathrm{Al}^{\mathrm{t}}+\mathrm{Mg}+\mathrm{Fe}^{2+}$ & 19.95 & 19.53 & & 19.91 & 19.50 & & 19.92 & 19.55 & & 19.95 & 19.91 & \\
\hline $\mathrm{Al}^{\mathrm{t}}+\mathrm{Mg}+\mathrm{Fe}^{2+}$ & 13.45 & 13.17 & & 13.63 & 13.35 & & 13.57 & 13.32 & & 14.16 & 14.13 & \\
\hline \multirow{3}{*}{$\begin{array}{l}\text { Sample } \\
n\end{array}$} & \multicolumn{3}{|c|}{ DG-68 } & \multicolumn{3}{|c|}{ Dt-8 (118.0m) } & \multicolumn{3}{|c|}{ Dt-8 (129.5m) } & & $8(207.8$ & \\
\hline & & 5 & & & 6 & & & 8 & & & 4 & \\
\hline & $x$ & & $s$ & $x$ & & $s$ & $x$ & & $s$ & $x$ & & $s$ \\
\hline $\mathrm{SiO}_{2}$ & 23.69 & & 0.33 & 23.9 & & 0.4 & 23.67 & & 0.49 & 26.81 & & 0.89 \\
\hline $\mathrm{TiO}_{2}$ & 0.09 & & 0.01 & 0.02 & & 0.02 & 0.06 & & 0.03 & 0.11 & & 0.06 \\
\hline $\mathrm{Al}_{2} \mathrm{O}_{3}$ & 21.3 & & 0.43 & 22.25 & & 0.66 & 20.98 & & 1.04 & 18.99 & & 1.06 \\
\hline${ }^{\circ} \mathrm{FeO}$ & 33.74 & & 0.74 & 31.26 & & 0.62 & 35.81 & & 1.16 & 20.25 & & 0.91 \\
\hline $\mathrm{MnO}$ & 0.42 & & 0.03 & 0.25 & & 0.03 & 0.07 & & 0.01 & 0.1 & & 0.02 \\
\hline $\mathrm{MgO}$ & 8.69 & & 0.24 & 9.42 & & 0.19 & 7.51 & & 0.65 & 17.1 & & 1.11 \\
\hline $\mathrm{CaO}$ & 0.04 & & 0.03 & 0.02 & & 0.01 & 0.05 & & 0.04 & 0.05 & & 0.03 \\
\hline $\mathrm{NaO}$ & 0.02 & & 0.02 & 0.11 & & 0.05 & 0.04 & & 0.04 & 0.1 & & 0.04 \\
\hline $\mathrm{K}_{2} \mathrm{O}$ & 0.01 & & 0.01 & 0.08 & & 0.04 & 0.04 & & 0.08 & 0.05 & & 0.03 \\
\hline Total & 88.00 & & 0.77 & 87.31 & & 0.89 & 88.23 & & 1.00 & 83.56 & & 3.44 \\
\hline & $x^{\mathrm{a}}$ & $x^{\mathrm{b}}$ & $s$ & $x^{\mathrm{a}}$ & $x^{\mathrm{b}}$ & $s$ & $x^{\mathrm{a}}$ & $x^{\mathrm{b}}$ & $s$ & $x^{\mathrm{a}}$ & $x^{\mathrm{b}}$ & $s$ \\
\hline $\mathrm{Si}$ & 5.24 & 5.23 & 0.08 & 5.28 & 5.24 & 0.09 & 5.29 & 5.27 & 0.14 & 5.85 & 5.78 & 0.16 \\
\hline $\mathrm{Al}^{\mathrm{IV}}$ & 2.76 & 2.77 & 0.08 & 2.72 & 2.76 & 0.09 & 2.71 & 2.73 & 0.14 & 2.15 & 2.23 & 0.16 \\
\hline $\mathrm{Al}^{\mathrm{t}}$ & 5.58 & 5.57 & & 5.79 & 5.75 & & 5.52 & 5.50 & & 4.88 & 4.82 & \\
\hline $\mathrm{Al}^{\mathrm{VI}}$ & 2.82 & 2.80 & 0.03 & 3.07 & 2.99 & 0.06 & 2.81 & 2.77 & 0.10 & 2.73 & 2.59 & 0.05 \\
\hline $\mathrm{Ti}$ & 0.01 & 0.01 & 0.00 & 0.00 & 0.00 & 0.00 & 0.01 & 0.01 & 0.01 & 0.02 & 0.02 & 0.01 \\
\hline $\mathrm{Fe}^{2+\mathrm{c}}$ & 6.24 & 6.23 & 0.11 & 5.77 & 5.73 & 0.11 & 6.68 & 6.66 & 0.20 & 3.69 & 3.65 & 0.07 \\
\hline $\mathrm{Mg}$ & 2.86 & 2.86 & 0.09 & 3.10 & 3.08 & 0.07 & 2.50 & 2.49 & 0.23 & 5.55 & 5.49 & 0.17 \\
\hline $\mathrm{Mn}$ & 0.08 & 0.08 & 0.01 & 0.05 & 0.05 & 0.01 & 0.01 & 0.01 & 0.00 & 0.02 & 0.02 & 0.00 \\
\hline Sum $\mathrm{R}^{\mathrm{VI}}$ & 12.01 & 11.98 & & 11.99 & 11.85 & & 12.01 & 11.94 & & 12.01 & 11.77 & \\
\hline $\mathrm{Ca}$ & 0.01 & 0.01 & 0.01 & 0.00 & 0.00 & 0.00 & 0.01 & 0.01 & 0.01 & 0.01 & 0.01 & 0.01 \\
\hline $\mathrm{Na}$ & 0.01 & 0.01 & 0.01 & 0.05 & 0.05 & 0.02 & 0.02 & 0.02 & 0.02 & 0.04 & 0.04 & 0.02 \\
\hline K & 0.00 & 0.00 & 0.00 & 0.02 & 0.02 & 0.01 & 0.01 & 0.01 & 0.02 & 0.02 & 0.02 & 0.01 \\
\hline $\operatorname{Sum}(\mathrm{Ca}+\mathrm{Na}+\mathrm{K})$ & 0.02 & 0.02 & & 0.07 & 0.07 & & 0.04 & 0.04 & & 0.07 & 0.07 & \\
\hline Total & 20.03 & 20.00 & & 20.06 & 19.92 & & 20.05 & 19.98 & & 20.08 & 19.84 & \\
\hline$X_{\mathrm{Mg}}$ & 0.24 & 0.24 & & 0.26 & 0.26 & & 0.21 & 0.21 & & 0.46 & 0.46 & \\
\hline $\mathrm{Fe}^{2+} /\left(\mathrm{Fe}^{2+}+\mathrm{Mg}\right)$ & 0.69 & 0.69 & & 0.65 & 0.65 & & 0.73 & 0.73 & & 0.40 & 0.40 & \\
\hline $\mathrm{Si} /\left(\mathrm{Si}+\mathrm{Al}^{\mathrm{t}}\right)$ & 0.48 & 0.48 & & 0.48 & 0.48 & & 0.49 & 0.49 & & 0.55 & 0.55 & \\
\hline $\mathrm{Si}+\mathrm{Al}^{\mathrm{t}}+\mathrm{Mg}+\mathrm{Fe}^{2+}$ & 19.92 & 19.89 & & 19.94 & 19.80 & & 19.99 & 19.92 & & 19.97 & 19.74 & \\
\hline $\mathrm{Al}^{t}+\mathrm{Mg}+\mathrm{Fe}^{2+}$ & 14.68 & 14.66 & & 14.66 & 14.56 & & 14.70 & 14.65 & & 14.12 & 13.96 & \\
\hline
\end{tabular}

$x=$ mean value; $s=$ standard deviation; $n=$ number of analyses. Cation numbers calculated on the basis of fixed cation sum: tetrahedral + octahedral cations $=20^{\mathrm{a}}$ and on the basis of 28 oxygens $s^{\mathrm{b}}{ }^{\mathrm{c}}$ Total $\mathrm{Fe}$ calculated as $\mathrm{Fe}^{2+}$.. 
which are separate from packets of illite or muscovite. Glycolation thus causes expansion of such layers, but with no or little effect on the illite or muscovite. The contribution of such layers to the $10 \AA$ peak of the XRD pattern thus is separated from the contribution of illite or muscovite, i.e. the contribution of mixed layering to the $10 \AA$ peak is largely eliminated by glycolation. On the other hand, expandable smectiteor vermiculite-like layers occur largely intercalated within packets dominated by chlorite. Since XRD averages the contributions over all parallel layers in a packet, expansion of these layers causes a small increase in average $d$ value and a larger range in $d$ values. The $14 \AA$ peak therefore becomes broader, and the mean position shifts to lower theta.

The XRD data show that the effect of glycolation diminishes with increasing grade (Table 4), consistent with a decrease in the proportions of expandable mixed layers with increasing metamorphic grade. Thus, the proportion of mixed layers in chlorite is one of the main factors that controls $\mathrm{ChC}$, especially $\mathrm{ChC}(001)$, in low-grade samples. This result confirms the statements of Yang \& Hesse (1991) and Árkai (1991) on the role of mixed layers in changes in $\mathrm{ChC}(001)$ in lower grade metapelites.

\section{XRD-based crystallite size vs. metamorphic grade}

Accurate determination of the absolute values of mean

crystallite size and lattice strain by analyses of XRD line profiles is hindered by numerous factors (Lanson \& Kübler, 1994; Árkai et al., 1996, 1997; Warr, 1996; Drits et al., 1997). Nevertheless, the results obtained by the Scherrer and Voigt methods (Tables 5-7) confirm that the apparent mean crystallite size of chlorite increases with increasing grade. Furthermore, apparent mean crystallite size is a principal factor influencing $\mathrm{ChC}(001)$ and $\mathrm{ChC}(002)$, especially at higher grades (higher anchizone and epizone), where the amounts of mixed layers are practically negligible or non-existent. The range of changes in apparent mean crystallite size of illite-muscovite is larger than that of chlorite. These relations are in accord with the earlier conclusions of Árkai \& Tóth (1990), Merriman et al. (1995) and Árkai et al. $(1996,1997)$.

In contrast to the regular variation in mean crystallite size, the XRD-calculated, apparent, mean lattice strain of chlorite, as calculated by the Voigt method (Tables $7 \& 8$ ), does not show systematic change as a function of metamorphic grade. However, the calculated lattice strain of illite-muscovite decreases with increasing grade (Table 6). Similar differences in the behaviour of chlorite and illite-muscovite during tectonic strain-induced recrystallization were described by Merriman et al. (1995) and Árkai et al. (1996, 1997).

As compared with the size data outlined above, the chlorite crystallite size data calculated by Krumm's WINFIT $2.1^{\circledR}$ program are generally smaller. Values obtained using the Warren-Averbach method do not follow the trend predicted from the $\mathrm{ChC}$ data (Table 9). Comparison of the results of the various XRD-based methods implies that the higher the metamorphic grade (i.e. the larger the mean crystallite size), the larger are the differences between the results of the various methods, even using the same natural sample as a 'standard'. This further demonstrates that such values are only apparent mean values, and should be used only for expressing and interpreting relative differences (see also Árkai et al., 1996, 1997). Nevertheless, the fairly good agreement found between the various methods for the diagenetic samples implies that they give at least realistic approximate measures of absolute values.

\section{TEM-measured crystallite size distributions}

Figure 3 shows histograms of crystal thickness as

determined from TEM images of ion-milled samples. The histograms for low-grade metapelites contain data only for authigenic/metamorphic grains; although detrital grains occur with sizes in the micrometre range and larger, we assume that such grains are largely or entirely excluded from the fractions used for XRD, and therefore are excluded from the histograms of TEM-determined values. If data for detrital grains were included, the histograms would have large populations with mean thicknesses $>>1 \mu \mathrm{m}$, largely separated from the populations of authigenic/metamorphic grains.

The distributions show two kinds of change with increasing grade. (1) The range of values increases dramatically. Most grains have sharply defined thicknesses $<500 \AA$ at low grades with a slight spread towards greater thicknesses, but distributions at high grades are spread over several thousand angstroms, with poorly defined maxima. (2) The mean sizes increase. During increasing regional (tectonically induced) metamorphism, various microstructural settings develop in metapelites (cleavage planes with high shear strain and high strain rate, intercleavage domains with low shear strain where there is spaced cleavage, etc.). Because strain causes a decrease in relative stability, differences in strain rates result in a range of local environments in which dissolution and subsequent neocrystallization and increase in size of existing crystals (ripening) occur. The result is great differences in crystallite sizes in various microstructural sites. Even greater differences in microstructural domains are found in the metabasites as a result of heterogeneity in the contrast in texture involving the matrix, large primary crystals, veins, vugs, etc. Such factors account both for the increase in range of crystallite thicknesses and in the mean (or median) thicknesses with increasing grade.

Although the forms of the crystal size distributions change regularly and predictably with increasing grade, some distributions are not well defined because of the small numbers, $n$, of crystallites counted. The frequency 
distributions of Fig. 3 show that larger numbers $(n)$ of crystallite size measurements are necessary for higher grade samples in order to define the broader ranges of crystallite thicknesses. As shown by the statistical parameters given in Fig. 3, even a value of $n$ as small as 100 is barely sufficient for defining reliable mean, modal and median values for the higher grade rocks. Specific mean, median or modal values are therefore relatively imprecise. Nevertheless, the general trends in values are well defined as a function of grade.

Comparison of the chlorite mean crystallite size data calculated by the Scherrer and Voigt methods on the $<2 \mu \mathrm{m}$ fractions, and the TEM mean values measured on ion-milled whole-rock samples, allows the following conclusions to be drawn (Fig. 3 and comparison with data of Tables $5,7 \& 8$ ).

1 For the lowest grade, prehnite-pumpellyite facies metabasite samples, fairly good agreement is found between the TEM-measured and XRD-calculated mean values.

2 The chlorite TEM mean values of the two diagenetic pelites are larger than the XRD-calculated values. Similar differences were found for illite-muscovite from pelitic rocks by Merriman et al. (1990), Lanson \& Kübler (1994), Jiang et al. (1997), Li et al. (1998) and Warr \& Nieto (1998).

3 The discrepancy between the XRD-calculated and TEM-measured mean crystallite size values of chlorite increases with increasing grade in both lithologies, all TEM-determined mean values being larger (in certain cases up to $c$. five to seven times) than the XRD values. Similar relations were found for illite-muscovite from metapelites by Jiang et al. (1997) and Li et al. (1998). One of the main causes of this relation considered by these authors was an increase in mechanical damage of crystallites during XRD sample preparation with increasing metamorphic grade, i.e. with increasing sample coherency as grains grow and become interlocked. The data of this study cast doubt on this conclusion, however. Although textural relations, and therefore breakage properties, change dramatically for metapelites, the textures of the metabasites are minimally changed with increasing grade.

A plausible cause of this discrepancy concerns the negligible effect of large $(c .>1000 \AA)$ crystallites on XRD line broadening and, consequently, the XRDcalculated mean size values, as explained by Klug \& Alexander (1974). The relatively large crystallites measured by TEM strongly influence the TEM mean values. In fact, the proportions of crystallites $<1000 \AA$ thick decrease markedly from $100 \%$ in diagenetic samples to c. $30 \%$ in epizonal (greenschist facies) samples. In addition, the proportion of the $<2 \mu \mathrm{m}$ fraction decreases with increasing ripening. These relations imply that the mean sizes of chlorite crystallites calculated from XRD line profiles obtained from the $<2 \mu \mathrm{m}$ grain size fractions are not characteristic of a given metamorphic sample as a whole, at least for those grades above the diagenetic zone and lower anchizone (see also Li et al., 1998).

\section{Evolution of chlorite composition}

Surprisingly, as shown in Fig. 4(a), EMP-determined average compositions of chlorite (Table 11) have significantly lower interlayer cation $(\mathrm{Na}+\mathrm{K}+\mathrm{Ca})$ contents than AEM results (Table 10). Interlayer cation contents are normally greater in EMP analyses because the EMP beam sample area is one to two orders of magnitude larger than that of AEM and therefore more likely to include other phases, and because the areas chosen for AEM analysis are normally first characterized as homogeneous by TEM imaging (Jiang et al., 1994; Essene \& Peacor, 1995).
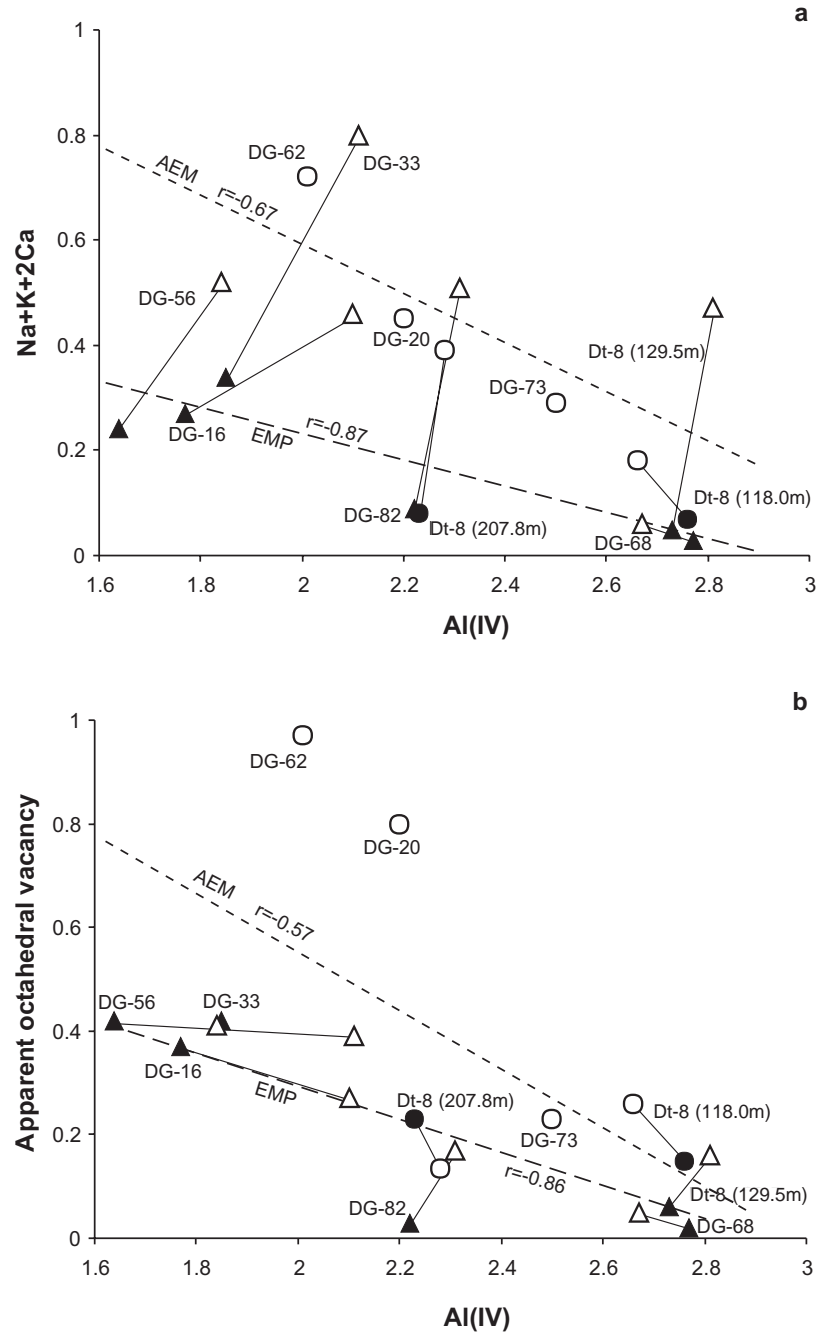

Fig. 4. Relations between the $\mathrm{Al}^{\mathrm{IV}}$ content and total interlayer charge expressed by $\mathrm{Na}+\mathrm{K}+2 \mathrm{Ca}$ values (a) and apparent octahedral vacancies (b) of chlorite. Apparent octahedral vacancies were calculated by the formula: $\square=20-[$ sum of the cations except $\mathrm{Na}, \mathrm{K}$ and $\mathrm{Ca}$. Cation numbers were calculated on the basis of 28 oxygen atoms. Circle, metapelite; triangle, metabasite. Open symbols, AEM mean values; filled symbols, EMP mean values. 
Although care was taken to obtain AEM analyses only from crystals first found to be as homogeneous as possible by observation of images, $\mathrm{Na}$ was almost always present only in analyses of chlorite from albitebearing rocks, implying the possibility of contamination during the ion-milling process, with atoms removed from one portion of the sample being deposited on other areas. Although rarely observed, this is the most likely source of differences in AEM and EMP analyses. Nevertheless, there are no systematic differences for other elements, the AEM and EMP data being similar for coeval pairs, and showing the same average trends.

Nevertheless, the total interlayer charge as given by the sum $(\mathrm{Na}+\mathrm{K}+2 \mathrm{Ca})$ and by the apparent octahedral vacancies of trioctahedral 'chloritic' material decrease with increasing tetrahedral $\mathrm{Al}$ content, i.e. with increasing metamorphic grade (Fig. 4b). This confirms the conclusions of Jiang et al. (1994) and many others (for reviews, see Alt, 1999; Schiffman \& Day, 1999) that the increase in $\mathrm{Al}^{\mathrm{IV}}$ content of chlorite with increasing grade is a consequence of decreasing amounts of mixed layers, primarily swelling mixed layers, rather than of increasing substitution of $\mathrm{Si}$ for $\mathrm{Al}$ in the wellordered mineral chlorite (see also Árkai \& Sadek Ghabrial, 1997).

The data plotted in Fig. 5, which illustrate the decrease in apparent octahedral vacancies and $\mathrm{Si}$ contents (i.e. increase in $\mathrm{Al}^{\mathrm{IV}}$ contents) with increasing metamorphic grade, demonstrate the evolution of chlorite relative to the content of mixed-layered material. The trends for chlorite of the metabasites imply changes in amounts of trioctahedral smectitic (saponitic) impurities. This is confirmed by the TEM observations, namely by the direct observation of up to $25 \%$ randomly intercalated $\mathrm{Mg}$-rich smectitic (or vermiculitic) layers, or by locally ordered corrensite. The chloritic materials of diagenetic pelites display compositional trends also consistent with TEM observations; in the latter case, however, the mixed layers are primarily of a berthierine-like $7 \AA$ phase. They occur both as discrete packets and as layers intercalated within chlorite packets. Considerable amounts of interlayer cations in chlorite of diagenetic pelites (samples DG-62, DG-20, see Fig. 4a) suggest that smectitic impurities that were not identified by TEM may also occur. The plotted points for higher grade chlorite along the line for which the apparent octahedral vacancy is minimal $(\mathrm{V}=0)$ in Fig. 5 are related to the various bulk rock compositions of the samples (see also Árkai \& Sadek Ghabrial, 1997).

The divergent trends of chlorite compositions caused by trioctahedral (saponitic) smectite impurities in metaigneous rocks and by berthierine and dioctahedral smectite in pelitic rocks are evidenced also by Fig. 6 . Figure 7 is a plot of the temperatures of formation calculated using Cathelineau's (1988) chlorite- $\mathrm{Al}^{\mathrm{IV}}$ geothermometer, but as determined as a function of percentage of chlorite in mixed-layered chlorite/smec-

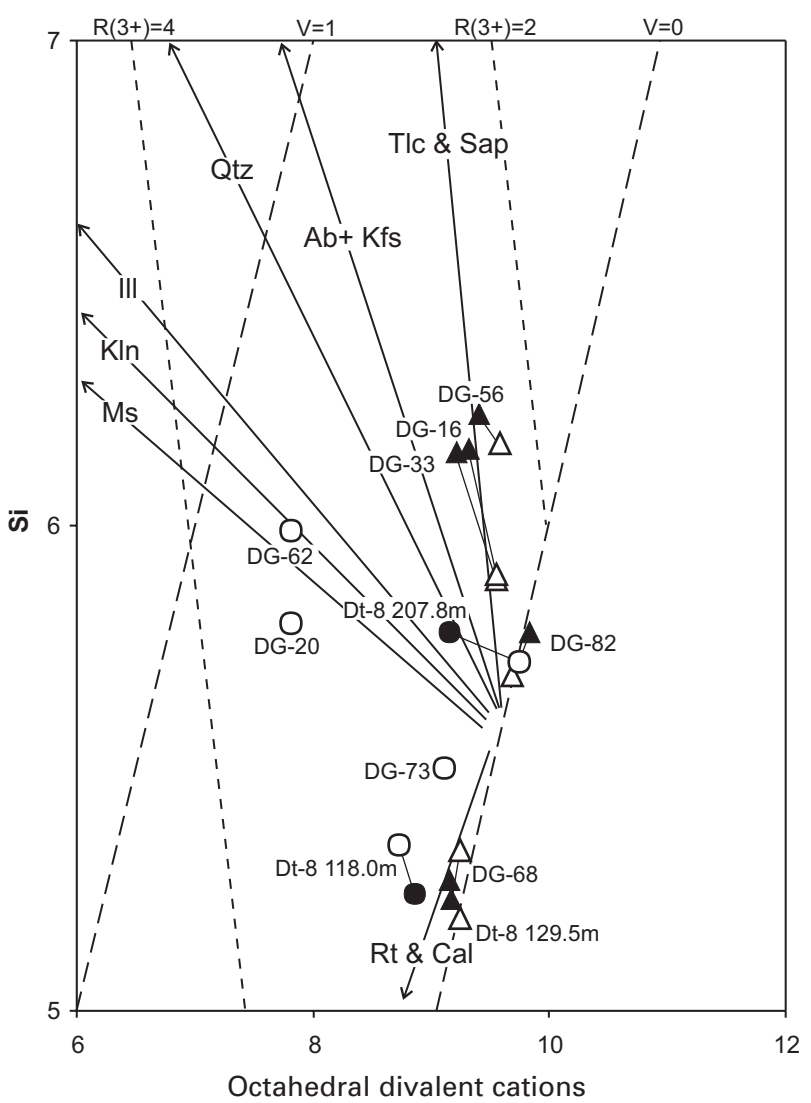

Fig. 5. Si vs. octahedral divalent cations plotted after Jiang et al. (1994). Arrows indicate schematic trends of compositional deviations generated from mixtures of an assumed composition of chlorite

$\left[\mathrm{Fe}_{5.5} \mathrm{Mg}_{4} \mathrm{Al}_{2.5}\left(\mathrm{Si}_{5.5} \mathrm{Al}_{2.5} \mathrm{O}_{20}\right)(\mathrm{OH})_{16}\right]$ and other minerals. V, apparent octahedral vacancies; $\mathrm{R}^{3+}$, octahedral divalent cations. For symbols, see Fig. 4. Abbreviations of mineral names after Bucher \& Frey (1994).

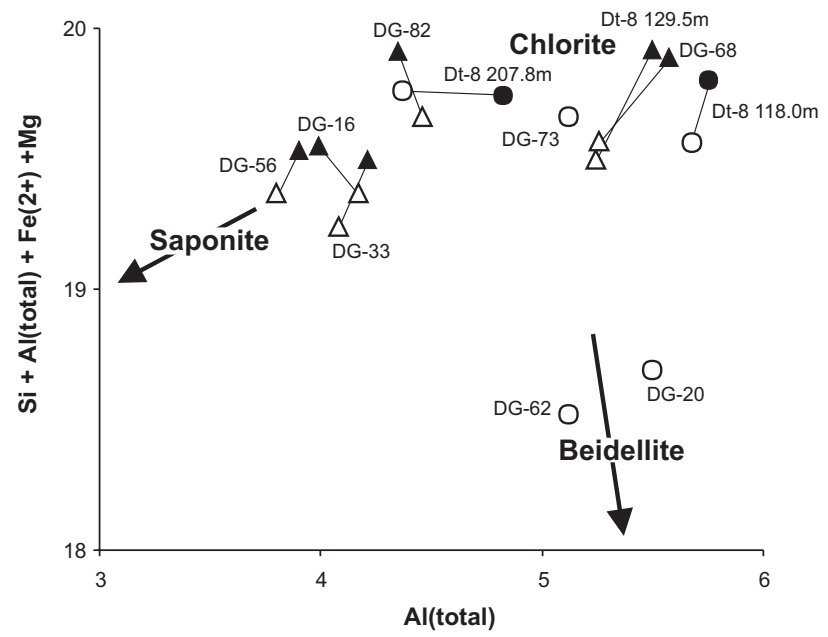

Fig. 6. Divergent trends in chlorite of metabasites (triangles) and metapelites (circles) as plotted after Schiffman \& Fridleifsson (1991). Open symbols, AEM mean values; filled symbols, EMP mean values. Cation numbers on the basis of 28 oxygen atoms. 


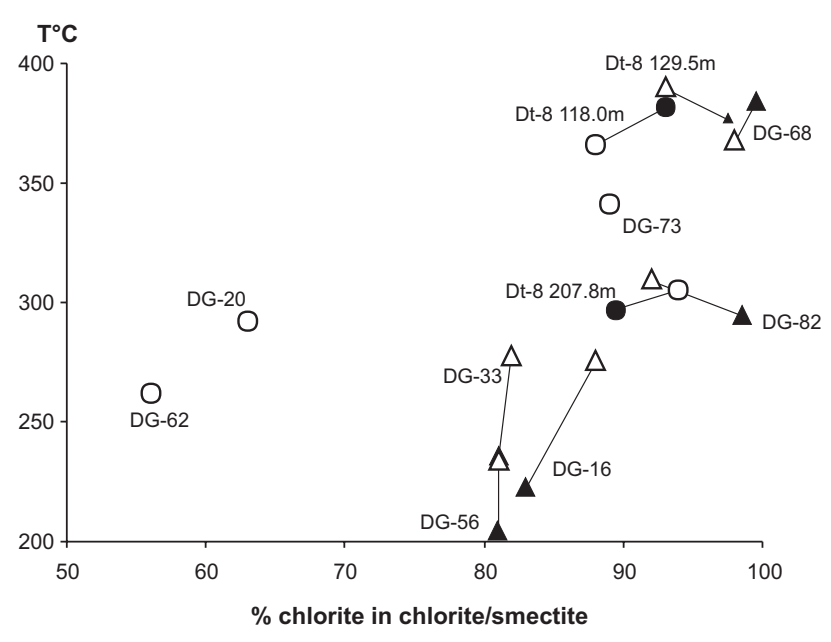

Fig. 7. Relation between the proportion of chlorite in mixedlayered chlorite/smectite estimated using the technique of Bettison \& Schiffman (1988) and temperatures calculated by the chlorite- $\mathrm{Al}^{\mathrm{IV}}$ geothermometer of Cathelineau (1988). See caption of Fig. 4 for symbols.

tite as defined by Bettison \& Schiffman (1988). This scheme uses the deviations of the non-interlayer cation sums of the measured phase from that of ideal trioctahedral chlorite, and determines the percentage chlorite value by linear interpolation between the cation sums of trioctahedral smectite and chlorite, both normalized to 28 oxygen atoms. This procedure results in minimum proportions of chlorite, because even minor octahedral vacancies in chlorite, sensu stricto, cause an apparent increase in the estimate of the smectitic component. The various impurities in chlorite are reflected also in the plotted data of Fig. 7. The anomalously low, unrealistic percentages of chlorite in chlorite/smectite of the diagenetic pelites DG-62 and DG-20 are related to the transitional berthierine and dioctahedral smectite components, as described above. By contrast, the estimated values for metabasites are in agreement with TEM observations which imply up to $25 \%$ mixed layering in chlorite. With increasing grade, the proportion of chlorite in chlorite/smectite increases from 81 to $99.5 \%$. There is an approximate, positive linear correlation between the percentage chlorite in chlorite/smectite and temperatures obtained by the $\mathrm{Al}^{\mathrm{IV}}$ thermometer, implying that the amounts of expandable mixed layers play an important role in the apparent $\mathrm{Al}^{\mathrm{IV}}$ contents of the analysed chlorite samples. Temperatures calculated for diagenetic pelites (DG-62 \& DG-20) are 262 and $292{ }^{\circ} \mathrm{C}$, respectively, $341{ }^{\circ} \mathrm{C}$ for the higher anchizonal slate (DG-73) and 366 and $305^{\circ} \mathrm{C}$ for the epizonal slate and metasandstone (Dt-8 (118.0m) \& Dt-8 $(207.8 \mathrm{~m})$ ), respectively, the diagenetic values being rather unrealistic. The prehnite-pumpellyite facies rocks gave temperature ranges of $202-234{ }^{\circ} \mathrm{C}$ (Darnó Hill) and $223-278^{\circ} \mathrm{C}$ (Szarvaskö complex), the pumpellyite-actinolite facies metabasite $295-310^{\circ} \mathrm{C}$ and the greenschist facies metabasites $377-390{ }^{\circ} \mathrm{C}$, the AEM data providing the higher estimates. The temperature ranges for the higher grade rocks seem to be reasonable as compared with the generalized facies schemes of Liou et al. (1987) and Bucher \& Frey (1994). Nevertheless, such temperatures are more properly interpreted as measures of reaction progress, as discussed below.

\section{Traces of retrogression as evidenced by TEM observations}

Rare $24 \AA$ chlorite/smectite mixed layers and berthierine $7 \AA$ layers were observed in TEM images of pumpellyite-actinolite and greenschist facies metabasites (DG-82, DG-68) and higher anchizonal slate (DG-73). Such mixed layering is generally observed only in low-grade rocks of prograde diagenetic and low-grade metamorphic sequences, for which it is a direct expression of non-equilibrium crystallization at low temperatures. These materials were observed only on the rims of grains which were otherwise homogeneous and of sizes consistent with relatively high grades, however. They are therefore inferred to have formed as retrograde material, subsequent to, and at lower temperatures than, peak metamorphism. Such retrograde effects have been observed recently in several cases (Merriman \& Peacor, 1999), and are inferred to occur commonly, although they generally may not be clearly identified through XRD patterns where the relative proportion of retrograde material is small. Nevertheless, such material may cause subtle broadening of diffraction peaks, and thus affect IC and ChC values and conclusions regarding crystallite thickness and metamorphic grade. In the present case, however, no such correlations could be discerned, the amounts of retrograde clays apparently being so small as to have no detectable effect on XRD patterns.

The retrogression can be attributed to mylonite formation in a shear zone of the Eastern Bükk, the effect of which is also shown by $\mathrm{K}-\mathrm{Ar}$ geochronology of K-white micas (Árkai et al., 1995a). Formation of berthierine, lateral transitions from one $14 \AA$ layer to two $7 \AA$ layers at chlorite rims, formation of corrensitic units in chlorite of greenschist facies metabasalt (Dt-8 $(129.5 \mathrm{~m}))$ and the occurrence of corrensite and random chlorite/smectite in epizonal metasandstone (Dt-8 (207.8m)) may be related to the migration of hydrothermal fluids subsequent to regional metamorphism (Árkai et al., 1981; Polgári \& Fórizs, 1996).

\section{Factors affecting chlorite crystallinity}

As evident from the Scherrer equation and as is demonstrated by the data plotted in Fig. 8(a), strong, hyperbolic negative correlation is found between ChC and XRD-calculated mean crystallite size for both $\mathrm{ChC}(001)$ and $\mathrm{ChC}(002)$. Similar relations exist between the $\mathrm{ChC}$ indices and TEM-measured mean crystallite size values (Fig. 8b). In contrast to mean size values, apparent mean lattice strain has no 

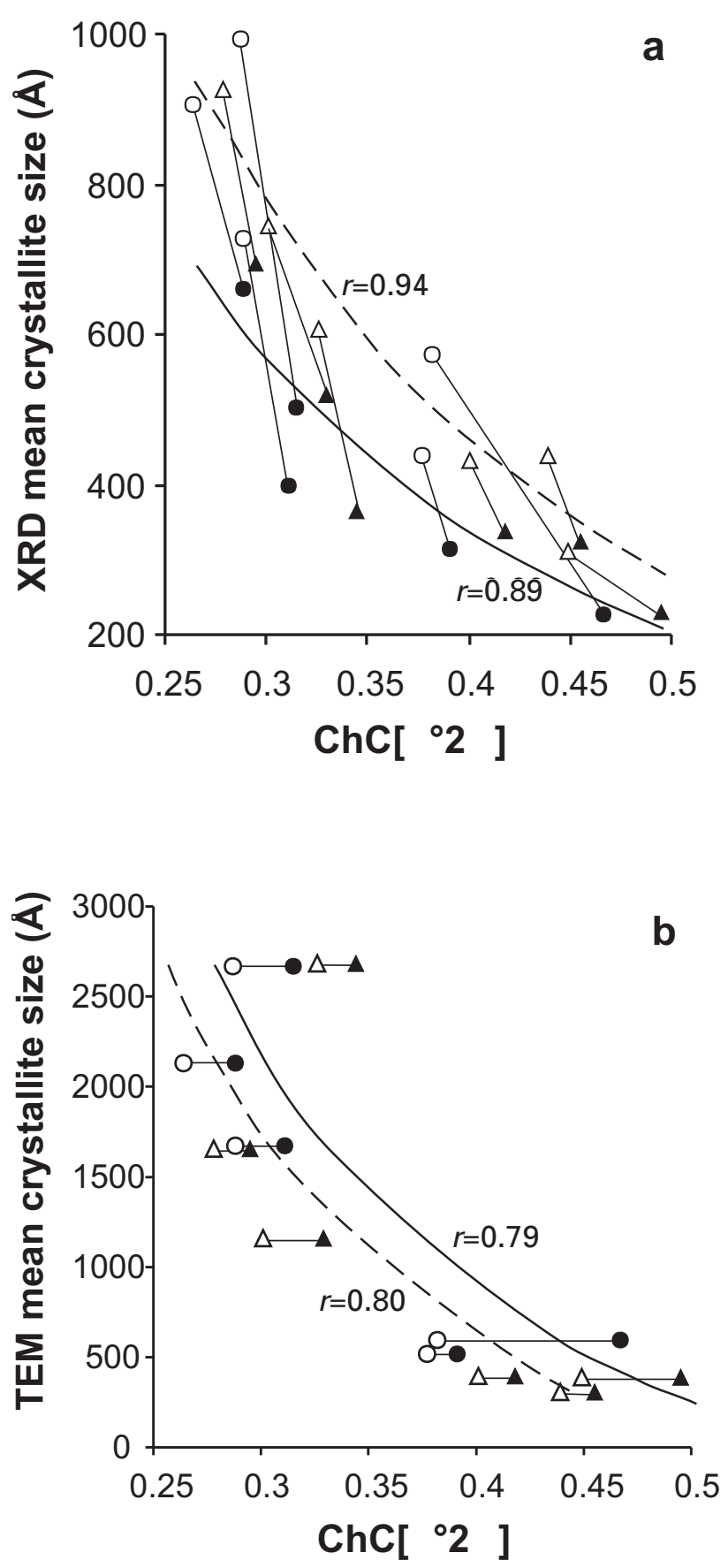

Fig. 8. Relations between chlorite crystallinity indices and apparent mean crystallite size determined by XRD-based Voigt method (a) and TEM measurements (b). Filled symbols, $\mathrm{ChC}(001)$; open symbols, $\mathrm{ChC}(002)$; triangles, metabasites; circles, metapelites. Regression lines and correlation coefficients for the function $y=a+b / x$ are also given.

appreciable effect on $\mathrm{ChC}$, most probably due to the fact that strain has only a minor influence on the shape of XRD line profiles at low diffraction angles (Klug \& Alexander, 1974).

In addition to mean crystallite size, the proportions of swelling (smectite- or vermiculite-like) mixed layers, which are present in small quantities even in the anchizonal sample, also strongly affect the $\mathrm{ChC}$ indices. This is in part shown by the linear positive correlations between $\mathrm{ChC}$ indices and $X_{\mathrm{Chl}}$ (Fig. 9a, b), total interlayer charge (Fig. 10a, b) and apparent octahedral vacancies (Fig. $11 \mathrm{a}, \mathrm{b}) . X_{\mathrm{Ch} 1}$ is strongly influenced also by the proportion of occupied octahedral sites. $X_{\mathrm{Chl}}$ can be used for estimating the ratio of smectite-like component only for ideal trioctahedral chlorite and smectite. Any deviations from this state result in overestimation of the smectite-like component in mixed-layer materials.

Largely because of decreasing amounts of smectitelike impurities in chlorite with increasing metamorphic grade, the calculated $\mathrm{Al}^{\mathrm{IV}}$ contents increase with decreasing $\mathrm{ChC}(001)$ and $\mathrm{ChC}(002)$ (Fig. 12a, b). Consequently, the $\mathrm{ChC}$ indices also correlate with the temperatures calculated by the Cathelineau (1988) so-called geothermometer. It must be emphasized that the interpretation of both the $\mathrm{Al}^{\mathrm{IV}}$ and the derived temperature values has considerably changed following the work of Jiang et al. (1994). Examples of such modified interpretations are found in the works of Árkai \& Sadek Ghabrial (1997), Schmidt \& Robinson (1997) and Schmidt et al. (1999). As reviewed by Merriman \& Peacor (1999), the so-called thermometer cannot be an accurate measure of absolute temperature because it is a measure of the proportions of metastable phases, which are a function of reaction kinetics and not in stable thermodynamic equilibrium. It is, however, a measure of reaction progress. The temperatures given for the samples of this study should not be interpreted as equivalent to absolute temperatures, therefore.

\section{CONCLUSIONS}

1 Values of $\mathrm{ChC}$ for pairs of coeval metabasites and metapelites are virtually identical, those for metapelites being only slightly smaller. The similarity occurs despite differences in kinds of mixed layering which represent metastable states, mostly berthierine layers in the case of metapelites and expandable smectitic or vermiculitic layers in metabasites.

$2 \mathrm{ChC}$ and IC values are reliable complementary tools for determining differences in metamorphic grade in a variety of rock types.

3 Collective XRD, TEM, AEM and EMP data for chloritic materials show that 'chlorite' crystallinity indices are controlled by: (i) the mean crystallite size of chlorite; and (ii) the proportion of random mixed layers and locally discrete intercalated phases in chlorite. Factor (ii) predominates at diagenetic grades and factor (i) almost exclusively at higher anchizonal and epizonal grades. By contrast, mean lattice strain, as determined by XRD-based methods, has no appreciable effect on ChC.

4 Differences in mean crystallite size values determined by various XRD-based calculation methods confirm 
Fig. 9. Chlorite crystallinity indices vs. proportion of chlorite in chlorite/smectite Schiffman, 1988). Filled symbols, continuous line, $X_{\mathrm{Chl}}$ from EMP analyses; open symbols, dashed line, $X_{\mathrm{Chl}}$ from AEM analyses. Triangle, metabasite; circle, metapelite.

Fig. 10. Chlorite crystallinity indices vs total interlayer charge of chlorites. For symbols, see Fig. 9.

Fig. 11. Chlorite crystallinity indices vs apparent octahedral vacancies measured by EMP and AEM. For symbols, see Fig. 9. (the latter calculated after Bettison \&
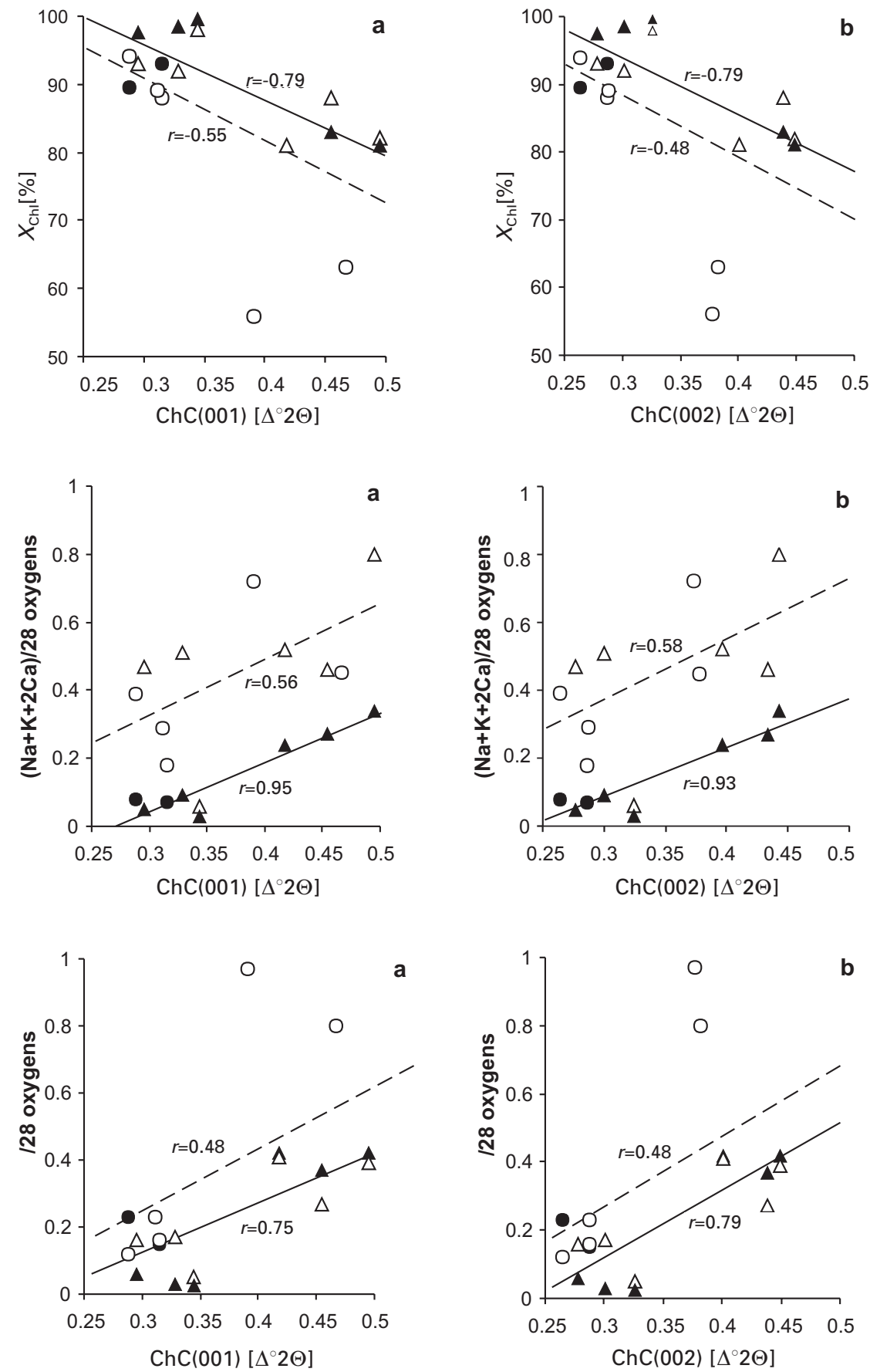

the earlier conclusions of Árkai et al. (1996, 1997), namely that these data can be considered only as apparent values. Data obtained by a given method using a given 'standard' for instrumental corrections can be used only for expressing relative differences, and should not be applied in an absolute sense. In general, differences between the results of XRD-based various calculation methods increase with increasing metamorphic grade.

5 The semiquantitative agreement between the TEMmeasured crystallite size distributions of chlorite for most of the metabasite-metapelite pairs gives further support to the conclusion that reaction progress is equivalent for both lithotypes.

6 The differences between the XRD-calculated and TEM-measured mean crystallite size values of chlorite increase with increasing grade. This discrepancy can be explained by the increasing proportions of large crystallites that are too thick to appreciably affect the sizes as calculated from XRD patterns. Thus, although XRD-based calculated results are very useful relative measures of crystallite thickness, the range of grade 


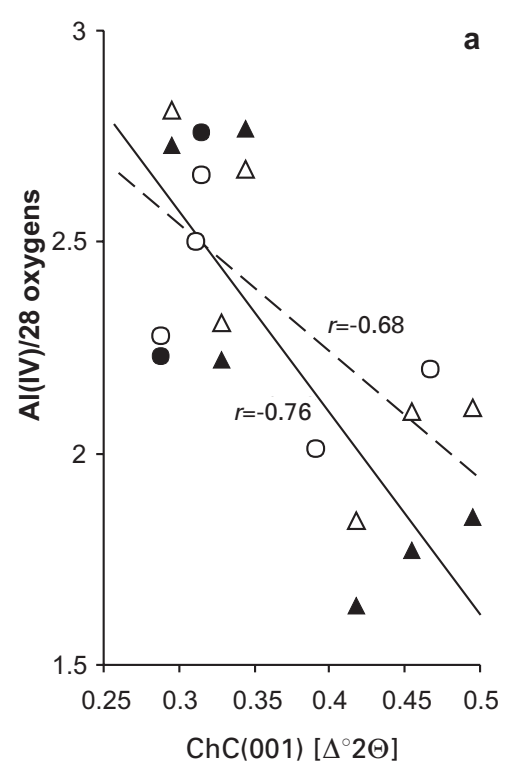

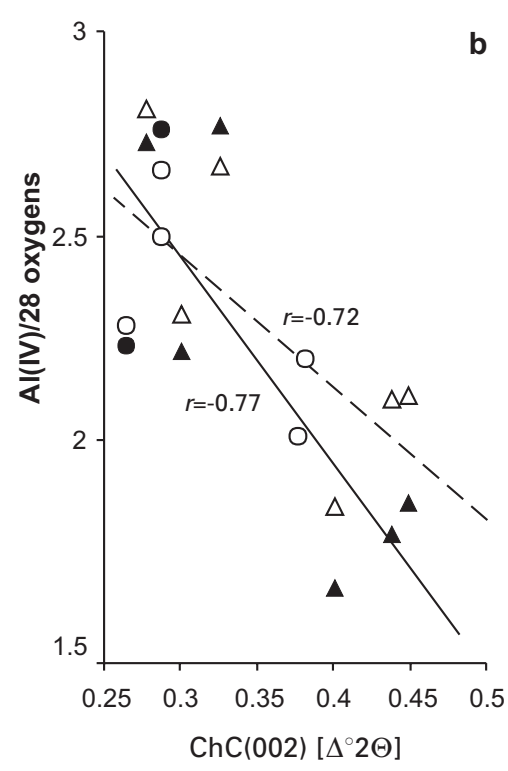

Fig. 12. Tetrahedral $\mathrm{Al}$ content of chlorite vs. chlorite crystallinity indices. For symbols, see Fig. 9. over which they give even approximate absolute values is limited. The ranges of chlorite crystallite size distributions become greater with increasing grade for both lithologies. Therefore, the number of the TEM size measurements required for correct characterization of size distribution is also a function of grade.

7 In contrast to XRD data that provide averaged information on rocks or on size fractions, TEM investigations of ion-milled whole-rock samples give new insights into the details of metamorphic and postmetamorphic reaction progress. Retrogression of phyllosilicates may be much more common than has generally been assumed.

As demonstrated by combined XRD, TEM, AEM and EMP studies, $\mathrm{ChC}$ is a powerful tool for metamorphic grade determination in rocks for which the application of conventional methods is difficult or impossible. Such rocks include, for example, metapelites containing 2:1 phyllosilicates having $d$ values of c. $10 \AA$ which interfere with contributions of illite, such as paragonite and/or margarite and/or pyrophyllite in addition to or instead of illite-muscovite, and metabasites and their clastic derivatives lacking key mineral assemblages. ChC, being a quantity which can be obtained easily, rapidly and with minimum expense, may have a rather large field of application for low-temperature metabasites; such rocks commonly comprise mineral assemblages such as quartz, albite, chlorite \pm carbonate minerals and various accessories which are not diagnostic measures of grade. Such mineral assemblages are stable over a rather large temperature range between $c$. $100-150$ and $400-450{ }^{\circ} \mathrm{C}$, were formed in the presence of $\mathrm{CO}_{2}$-containing fluids, and are widespread in ancient continental shelf environments. Thus the Palaeozoic and Mesozoic sequences of the Tethyan realm frequently contain such metaigneous and volcanoclastic rocks. $\mathrm{ChC}$ is thus an important tool in deciphering metamorphic conditions of such formations, for which the metamorphic history has previously been ill defined. ChC may also serve as a bridge between the illite crystallinity-based zonal classification of metapelites and the mineral facies system of metabasic rocks, as chlorite is a common rock-forming mineral in both lithologies.

\section{ACKNOWLEDGEMENTS}

The present work forms a part of the metamorphic petrological research programme of P.Á. sponsored by the Hungarian National Research Fund (OTKA, Budapest), Project No. T-022773/1997-2000. The authors are indebted to Dr. G. Nagy, Mr. Z. Wieszt and Ms. V. Varga (Laboratory for Geochemical Research, Hungarian Academy of Sciences) for the electron microprobe and major element bulk chemical analyses, and C. Henderson of the University of Michigan for help in TEM/AEM studies. Thanks are due to Mrs. O. Komoróczy, P. M. Sándor, Ms. K. Temesvári and N. Keresztes for their technical assistance and to Mr. P. Horváth for the graphical work. This research was supported by NSF grant EAR-98-14391 to D.R.P.

\section{REFERENCES}

Alt, J. C., 1999. Very low-grade hydrothermal metamorphism of basic igneous rocks. In: Low-Grade Metamorphism (eds Frey, M. \& Robinson, D.), pp. 169-226. Blackwell Science, Oxford. Árkai, P., 1983. Very low- and low-grade Alpine regional metamorphism of the Paleozoic and Mesozoic formations of the Bükkium, NE-Hungary. Acta Geologica Hungarica, 26, 83-101.

Árkai, P., 1991. Chlorite crystallinity: an empirical approach and correlation with illite crystallinity, coal rank and mineral facies as exemplified by Palaeozoic and Mesozoic rocks of northeast Hungary. Journal of Metamorphic Geology, 9, 723-734

Árkai, P., Balogh, K. \& Dunkl, I., 1995a. Timing of lowtemperature metamorphism and cooling of the Paleozoic and 
Mesozoic formations of the Bükkium, innermost Western Carpathians, Hungary. Geologische Rundschau, 84, 334-344.

Árkai, P., Balogh, K. \& Frey, M., 1997. The effects of tectonic strain on crystallinity, apparent mean crystallite size and lattice strain of phyllosilicates in low-temperature metamorphic rocks. A case study from the Glarus overthrust, Switzerland. Schweizerische Mineralogische und Petrographische Mitteilungen, 77, 27-40.

Árkai, P., Horváth, Z. A. \& Tóth, M., 1981. Transitional very low- and low-grade regional metamorphism of the Paleozoic formations, Uppony Mountains, NE-Hungary: mineral assemblages, illite-crystallinity, $-b_{o}$ and vitrinite reflectance data. Acta Geologica Academiae Scientarum Hungaricae, 24, 265-294.

Árkai, P., Merriman, R. J., Roberts, B., Peacor, D. R. \& Tóth, M., 1996. Crystallinity, crystallite size and lattice strain of illite-muscovite and chlorite: comparison of XRD and TEM data for diagenetic to epizonal pelites. European Journal of Mineralogy, 8, 1119-1137.

Árkai, P. \& Sadek Ghabrial, D., 1997. Chlorite crystallinity as an indicator of metamorphic grade of low-temperature metaigneous rocks: a case study from the Bükk Mountains, northeast Hungary. Clay Minerals, 32, 205-222.

Árkai, P., Sassi, F. P. \& Sassi, R., 1995b. Simultaneous measurements of chlorite and illite crystallinity: a more reliable geothermometric tool for monitoring low- to very low-grade metamorphism in metapelites. A case study from Southern Alps (NE. Italy). European Journal of Mineralogy, 7, 1115-1128.

Árkai, P. \& Tóth, M., 1990. Illite and chlorite 'crystallinity' indices, I: an attempted mineralogical interpretation. Abstract. Conference on 'Phyllosilicates as Indicators of Very Low-grade Metamorphism and Diagenesis' (IGCP 294), Manchester.

Bence, A. E. \& Albee, A., 1968. Empirical correction factors for electron microanalysis of silicates and oxides. Journal of Geology, 76, 382-403.

Bettison, L. A. \& Schiffman, P., 1988. Compositional and structural variations of phyllosilicates from the Point Sal ophiolite, California. American Mineralogist, 73, 62-76.

Bucher, K. \& Frey, M., 1994. Petrogenesis of Metamorphic Rocks, 6th edn. Springer-Verlag, Berlin.

Cathelineau, M., 1988. Cation site occupancy in chlorites and illites as a function of temperature. Clay Minerals, 23, 471-485.

Downes, H., Pantó, Gy., Arkai, P. \& Thirlwall, M. F., 1990. Petrology and geochemistry of Mesozoic igneous rocks, Bükk Mountains, Hungary. Lithos, 24, 201-215.

Drits, V., Srodon, J. \& Eberl, D. D., 1997. XRD measurement of mean crystallite thickness of illite and illite/smectite: reappraisal of the Kubler index and the Scherrer equation. Clays and Clay Minerals, 45, 461-475.

Essene, E. J. \& Peacor, D. R., 1995. Clay mineral thermometry - a critical perspective. Clays and Clay Minerals, 43, 540-553.

Frey, M., 1987. Very low-grade metamorphism of clastic sedimentary rocks. In: Low Temperature Metamorphism (ed. Frey, M.), pp. 9-58. Blackie, Glasgow \& London.

Jiang, W.-T., Peacor, D. R., Merriman, R. J. \& Roberts, B., 1990. Transmission and analytical electron microscopic study of mixed-layer illite/smectite formed as an apparent replacement product of diagenetic illite. Clays and Clay Minnerals, 38, 449-468.

Jiang, W.-T., Peacor, D. R. \& Buseck, P. R., 1994. Chlorite geothermometry? - contamination and apparent octahedral vacancies. Clays and Clay Minerals, 42, 593-605.

Jiang, W.-T., Peacor, D. R., Árkai, P., Tóth, M. \& Kim, J. W., 1997. TEM and XRD determination of crystallite size and lattice strain as a function of illite crystallinity in pelitic rocks. Journal of Metamorphic Geology, 15, 267-281.

Klug, H. P. \& Alexander, L. E., 1974. X-Ray Diffraction Procedures, 2nd edn. John Wiley, New York.

Kovács, S., 1989. Major events of the tectono-sedimentary evolution of the North Hungarian Paleo-Mesozoic: history of the northwestern termination of the Late Paleozoic-Early Mesozoic Tethys. In: Tectonic Evolution of the Tethyan
Region (ed. Sengor, A. M. C.), pp. 93-108. Kluwer Academic Publishers, Dordrecht

Kovács, S., Szederkényi, T., Árkai, P., Buda, Gy., Lelkes-Felvári, Gy. \& Nagymarosi, A., 1996-97. Explanation to the terrane map of Hungary. Annales Géol. Des Pays Helléniques, 37, 271-330.

Krumm, S., 1994. WINFIT1.0 - a public domain program for interactive profile-analysis under WINDOWS. XIIIth Conference on Clay Mineralogy and Petrology, Prague, 1994. Acta Universitatis Carolinae, Geologica, 38, 253-261.

Kübler, B., 1967. La cristallinité de l'illite et les zones tout a fait superieures du métamorphisme. In: Étages Tectoniques, Colloque de Neuchâtel 1966, pp. 105-121. Université Neuchâtel, A La Baconniere, Neuchâtel.

Kübler, B., 1968. Evaluation quantitative du métamorphisme par la cristallinité de l'illite. Bulletin du Centre de Recherches de Pau - SNPA, 2, 385-397.

Langford, J. I., 1978. A rapid method for analysing the breadth of diffraction and spectral lines using the Voigt function. Journal of Applied Crystallography, 11, 10-14.

Lanson, B. \& Kübler, B., 1994. Experimental determinations of the coherent scattering domain size distribution of natural mica-like phases with the Warren-Averbach technique. Clays and Clay Minerals, 42, 489-494.

Li, G. J., Peacor, D. R., Buseck, P. \& Árkai, P., 1998. Modification of illite-muscovite crystallite-size distributions by sample preparation for powder XRD analysis. Canadian Mineralogist, 36, 1435-1451.

Liou, J. G., Maruyama, S. \& Cho, M., 1987. Very low-grade metamorphism of volcanic and volcanoclastic rocks - mineral assemblages and mineral facies. In: Low Temperature Metamorphism (ed. Frey, M.), pp. 59-113. Blackie, Glasgow \& London.

Merriman, R. J. \& Frey, M., 1999. Patterns of very lowgrade metamorphism in metapelitic rocks. In: Low-Grade Metamorphism (eds Frey, M. \& Robinson, D.), pp. 61-107. Blackwell Science, Oxford.

Merriman, R. J. \& Peacor, D. R., 1999. Very low-grade metapelites: mineralogy, microfabrics and measuring reaction progress. In: Low-Grade Metamorphism (eds Frey, M. \& Robinson, D.), pp. 10-60. Blackwell Science, Oxford.

Merriman, R. J., Roberts, B. \& Peacor, D. R., 1990. A transmission electron microscope study of white mica crystallite size distribution in a mudstone to slate transitional sequence, North Wales, UK. Contributions to Mineralogy and Petrology, 106, 27-40.

Merriman, R. J., Roberts, B., Peacor, D. R. \& Hirons, S. R., 1995. Strain-related differences in the crystal growth of white mica and chlorite: a TEM and XRD study of the development of pelite microfabrics in the Southern Uplands thrust terrane, Scotland. Journal of Metamorphic Geology, 13, 559-576.

Polgári, M. \& Fórizs, I., 1996. Distribution of Mn in carbonates from the Uppony Mts., NE-Hungary. Geologica Carpathica, $47,215-225$.

Robinson, D. \& Bevins, R. E., 1999. Patterns of regional low-grade metamorphism in metabasites. In: Low-Grade Metamorphism (eds Frey, M. \& Robinson, D.), pp. 143-168. Blackwell Science, Oxford.

Robinson, D. \& Merriman, R. J., 1999. Low-temperature metamorphism: an overview. In: Low-Grade Metamorphism (eds Frey, M. \& Robinson, D.), pp. 1-9. Blackwell Science, Oxford.

Sadek Ghabrial, D., Árkai, P. \& Nagy, G., 1996. Alpine polyphase metamorphism of the ophiolitic Szarvaskö complex, Bükk Mountains, Hungary. Acta Mineralogia et Petrographia Szegediensis, 37, 99-128.

Schiffman, P. \& Day, H. W., 1999. Petrological methods for the study of very low-grade metabasites. In: Low-Grade Metamorphism (eds Frey, M. \& Robinson, D.), pp. 108-142. Blackwell Science, Oxford.

Schiffman, P. \& Fridleifsson, G. O., 1991. The smectite-chlorite transition in drillhole NJ-15, Nesjavellir geothermal field, Iceland: XRD, BSE, and electron microprobe investigations. Journal of Metamorphic Geology, 9, 679-696. 
Schmidt, D., Livi, K. J. T. \& Frey, M., 1999. Reaction progress in chloritic mineral: an electron microbeam study of the Taveyanne greywacke, Switzerland. Journal of Metamorphic Geology, 17, 229-241.

Schmidt, S. T. \& Robinson, D., 1997. Metamorphic grade and porosity and permeability controls on mafic phyllosilicate distributions in a regional zeolite to greenschist facies transition of the North Shore Volcanic Group, Minnesota. Geological Society of America Bulletin, 109, 683-697.

Teichmüller, M., 1987. Organic material and very low-grade metamorphism. In: Low Temperature Metamorphism (ed. Frey, M.), pp. 114-161. Blackie, Glasgow \& London.
Warr, L. N., 1996. Standardized clay mineral crystallinity data from the very low-grade metamorphic facies rocks of southern New Zealand. European Journal of Mineralogy, 8, 115-127.

Warr, L. N. \& Nieto, F., 1998. Crystallite thickness and defect density of phyllosilicates in low-temperature metamorphic pelites: a TEM and XRD study of clay mineral crystallinity index standards. Canadian Mineralogist, 36, 1453-1474.

Yang, C. \& Hesse, R., 1991. Clay minerals as indicators of diagenetic and anchimetamorphic grade in an overthrusted belt, external domain of southern Canadian Appalachians. Clay Minerals, 26, 211-231.

Received 9 January 2000; revision accepted 27 March 2000. 